\title{
Two-Dimensional Gravities and Supergravities as Integrable Systems
}

\author{
H. Nicolai \\ II. Institut für Theoretische Physik, \\ Universität Hamburg, \\ Luruper Chaussee 149, 2000 Hamburg 50, F.R.G.
}

\section{Introduction}

The study of exact solutions of Einstein's field equations in general relativity has a long history (for a comprehensive account of the subject, see [1]. A feature common to all such solutions is their independence of certain coordinates, or, in more technical terms, the existence of one or more Killing vectors. This independence is, first of all, important from a practical point of view because one would like to simplify the structure of the equations so as to make them amenable to explicit solution. In a more general perspective, the omission of two of the coordinates can be regarded as a special case of the Kaluza Klein program [2]. To study the solutions with two Killing vectors in a systematic fashion, one considers a Kaluza Klein type reduction of Einstein's theory to two dimensions, where the gravitational degrees of freedom are split into a "matter sector" describing the two physical helicity degrees of freedom and the remaining unphysical (non-propagating) degrees of freedom, in particular the conformal factor. The complications inherent in Einstein's theory are best illustrated by the long time it took to realize that the existence of exact solutions in this reduction is not just a fortuitous and accidental property of the theory, but intimately related to the existence of hidden symmetries and of certain infinitesimal transformations, which connect different solutions and which can be used to construct new solutions from old ones [3]. An important breakthrough occurred in 1970 , when Geroch was able to demonstrate that this set of transformations is, in fact, infinite-dimensional [4] (the corresponding group is nowadays referred to as the "Geroch group"). This was the first indication that Einstein's theory is actually integrable after the reduction to two dimensions. The idea was further developed and elaborated by the general relativists in the years following this discovery, with special emphasis on the search for "solution-generating procedures" [1,5-10]. This program has met with considerable success, especially with regard to the so-called stationary axisymmetric solutions possessing one space-like and one time-like Killing vector. The integrability of Einstein's equations was explicitly demonstrated with the 
construction of a linear system (or Lax pair) for the gravitational field equations by Belinskii and Sakharov [7] and Maison [8]. Thus, it became possible to apply techniques such as the inverse scattering method from the theory of integrable systems to Einstein's theory. There are, however, important differences between Einstein's theory and ordinary integrable systems: for instance, solutions of the gravitational field equations define their own domain of validity, whereas the space-time supporting the solution is a priori given for flat space integrable systems. This peculiar feature is reflected in the $x$-dependence of the spectral parameter entering the linear system $[7,8,10]$. Solutions describing colliding gravitational plane waves with two space-like Killing vectors have come into focus only much more recently [11], and the application of the solution-generating methods described above to this type of solutions is still in its infancy $[12,13]$.

Quite independently of these developments, particle physicists had become interested in Kaluza Klein theories and higher dimensional supergravities in the late seventies in their attempts to formulate a unified theory of the fundamental interactions (see e.g. [14]). One of the most remarkable developments in this context was the discovery of "hidden symmetries" in dimensionally reduced supergravities, culminating in the discovery of a hidden $E_{7}$ invariance in the maximally extended $N=8$ supergravity theory [15]. Apparently, B. Julia was the first to realize the connection between these developments and the work of the general relativists [16]. He emphasized the importance of group theoretical concepts for the investigation of the structural properties of dimensionally reduced gravity and supergravity theories and was led to conjecture that the emergence of infinite-dimensional symmetries in the reduction to two dimensions is a generic phenomenon. He was further able to show quite explicitly that the Geroch group in infinitesimal form is nothing but the affine Kac Moody algebra $A_{1}^{(1)}$ and to demonstrate the presence of a central term, which had gone unnoticed by the general relativists, and which acts as a shift operator on the conformal factor [17]. The underlying group theoretic structure and the connection with the $\sigma$-models encountered in particle physics were further elucidated by Breitenlohner and Maison $[10,18]$. They discovered a beautiful cocycle formula explicitly solving the equation of motion for the conformal factor. Through this work it has become clear that one can think of the space of solutions as some kind of infinite-dimensional coset space, which is analogous to the finite-dimensional coset spaces of gauge-equivalent field configurations in ordinary $\sigma$-models. It is, of course, crucial here that many concepts can be generalized from the finite-dimensional models to the infinite-dimensional case.

As for the purely bosonic models, these developments have been amply documented, and I will therefore try to avoid reduplication of the existing literature in these lectures by concentrating on those aspects which have not been given so much attention until now, and by shifting the emphasis from the systematics of solution-generating procedures to the discussion of group theoretical aspects and the search for yet bigger symmetries. This point of view is of more concern to the particle physicist and string theorist (and was already 
adopted in [16]). Nonetheless, I will also describe some concrete applications of the solution-generating procedures in section 3.4, where a special colliding plane wave solution of the Ferrari-Ibanez type [12] is reconstructed via the monodromy matrix. Special attention will be given to the fermionic models in these lectures. Although many results from the bosonic theory carry over to supergravity (such as the existence of a linear system yielding the supergravitational field equations $[19,20]$ ), there remain some puzzling questions which will be discussed at the end of these lectures. There is some evidence (to be presented in section 4.3) that there is more to the addition of fermions than just the popular recipe of turning every item into a super-item. For instance, the bosonic sector of the maximally extended $N=16$ theory in three dimensions [21] is described by an $E_{8} / S O(16)$ coset space non-linear $\sigma$-model, the two-dimensional reduction of which possesses a non-linearly realized $E_{9}$ symmetry independently of whether there are fermionic degrees of freedom or not. Contrary to one's naive expectations, the symmetry of the fully supersymmetric theory cannot be some ordinary supergroup, as $E_{8}$ has no supergroup extension [22]! Nonetheless, the symmetry must be enlarged in some way as there appears to be "no room" for the fermionic solutions in the bosonic coset spaces. The possibility that the required extension leads to hyperbolic algebras [23], which was already raised in [24], is discussed in section 4.3.

The search for more symmetries in the context of low dimensional supergravities could also provide some much needed hints for the formulation of a proper theory of quantum gravity, which is likely to be as much a problem in finding the correct underlying symmetry as in finding the correct conceptual framework. Let me briefly explain why it is that the study of $d=2$ or even $d=1$ reductions should afford any insight into this difficult problem. One reason for this expectation is what I would like to refer to (somewhat loosely) as the "string magic", by which an essentially trivial two-dimensional field theory is converted into a complicated higher-dimensional theory that contains (super)gravity as a tiny subsector. This is accomplished by re-interpreting the physical states of the theory on the two-dimensional world sheet as the oneparticle states of a quantum field theory in a higher-dimensional target space. This basic idea has been realized so far only in the context of conformally invariant theories, but has not been tried for the kind of theories we are about to discuss. Let us suppose that this is actually possible. Then, the Geroch group, which previously acted upon the space of solutions of a classical field equation, becomes a symmetry on the Hilbert space of physical quantum states defined through the imposition of the canonical constraints. Re-interpreting this space as a space of one-particle states of a higher-dimensional theory, one realizes that the quantum analog of the Geroch group now acts as a spectrum generating symmetry: it transforms the physical states into one another and possibly generates all of them from some groundstate (if it acts transitively). This spectrum generating algebra is reminiscent of an analogous spectrum generating algebra in string theory, which consists of the physical vertex operators [25] (a vertex operator is called physical if it weakly commutes with the Virasoro constraints). These similarities will be further illuminated in section 2.3. The point 
I would like to emphasize here is that the Geroch group has been converted from an abstract group acting on some equally abstract space of solutions into a "space-time symmetry" in this fashion. Since this symmetry constitutes a huge extension of the ordinary space-time symmetries, it may well be related to the elusive fundamental symmetry that string theorists have been searching for in vain until now.

There are also some interesting geometrical aspects which will be discussed in chapter 4 . In the formulation of $d=2$ (super)gravity as an integrable system described in chapter 3 , the basic object of interest is a matrix $\hat{\mathcal{V}}$ depending not only on the space-time coordinates but also on a so-called spectral parameter $t$. This matrix appears in the linear system (cf. section 3.3), and contains all the information about the equations of motion, including the fermionic ones in the case of supergravity. It may be viewed as an infinite-dimensional generalization of the vielbein in gravity. This analogy is suggested by the fact that the symmetry groups acting on this " $\infty$-bein" are in one-to-one correspondence with the groups acting on the ordinary vielbein: there is an infinite group $G^{\infty}$ (the Geroch group) acting rigidly on $\widehat{\mathcal{V}}$ from the left and another $H^{\infty}$ acting as a gauge group from the right. These groups contain the transverse subgroups of the corresponding finite-dimensional groups, namely $S L(D-2, \mathbb{R})$ and the transverse Lorentz group $S O(D-2)$. (This is the reason why I referred to the quantum Geroch group as a "space-time symmetry" above). The construction of even bigger symmetry groups involving "boost generators" will most probably require the further reduction to one dimension and the introduction of fermions.

Since the reduction to one dimension may be of interest in its own right, some pertinent results concerning maximal supergravity in one dimension are collected in the appendix. A novel feature is the proposal to perform the reduction with respect to one of the light-cone coordinates $x^{+}$or $x^{-}$, rather than $x^{0}$ or $x^{1}$. The potentially most important reason in favor of such a chiral (or "unidexterous") reduction is the possibility that it may give rise to a new kind of dual model, as I will briefly explain at the end of the appendix.

\section{Preliminaries}

This chapter summarizes some well-known results about the hidden symmetries of Einstein's theory in three space-time dimensions. As this subject has already been extensively dealt with in the literature [1], the presentation will be cursory and mainly serve to set up the notation. The results for $N=2$ supergravity in three dimensions are also given, because this is the simplest supersymmetric model, where the emergence of infinite-dimensional symmetries in the reduction to two dimensions can be studied. The main reason for the emphasis on three-dimensional Lagrangians here is that, in contradistinction to other approaches, I will always take the three-dimensional non-linear $\sigma$ model 
as the point of departure, thus bypassing the labor of explicitly working out the Kaluza Klein reduction from higher dimensions. Some salient features of the canonical treatment of hidden symmetries, which has been largely ignored in the relativists' literature, will be briefly discussed in section 2.3. (For further details concerning canonical gravity the reader is advised to consult the standard references and Isham's lectures in this volume). The relevance of hidden symmetries for the construction of non-trivial observables in canonical gravity is pointed out.

\subsection{The Reduction of Einstein's Theory to $d=3$}

The simplest example of a hidden symmetry is obtained when one reduces Einstein's theory from $d=4$ to $d=3$. After the reduction and a duality redefinition of the Kaluza Klein vector field, the theory possesses a hidden $S L(2, \mathbb{R})$ symmetry, the two physical gravitational degrees of freedom being parametrized by the $S L(2, \mathbb{R}) / S O(2)$ coset space. This fact is, of course, implicit in the earlier works on the subject. The presentation here follows [16,17]; the general case (e.g. Maxwell-Einstein) is discussed in [26], where further references can be found. The dimensional reduction simply amounts to dropping the dependence on the fourth coordinate for all fields $\phi$, viz.

$$
\phi\left(x^{0}, x^{1}, x^{2}, x^{3}\right) \longrightarrow \phi\left(x^{0}, x^{1}, x^{2}\right)
$$

This applies in particular for the vierbein, for which, in addition, we make partial use of the local $S O(1,3)$ invariance invariance to fix a triangular gauge, viz.

$$
E_{M}^{A}=\left(\begin{array}{cc}
\Delta^{-1 / 2} e_{m}^{a} & B_{m} \Delta^{1 / 2} \\
0 & \Delta^{1 / 2}
\end{array}\right)
$$

where the indices on the left hand side refer to four dimensions, while $m, \ldots=$ $0,1,2$ and $a, \ldots=0,1,2$ on the right hand side refer to three dimensions, and the extra factors of $\Delta$ have been inserted for convenience. Writing out Einstein's action in terms of

$$
F_{m n}=\partial_{m} B_{n}-\partial_{n} B_{m}
$$

we obtain

$$
\begin{aligned}
\mathcal{L}= & -\frac{1}{4} E R(E) \\
= & -\frac{1}{4} e R^{(3)}(e)+\frac{1}{8} e g^{m n} \Delta^{-1} \partial_{m} \Delta \Delta^{-1} \partial_{n} \Delta- \\
& -\frac{1}{16} e \Delta^{2} g^{m p} g^{n q} F_{m n} F_{p q}
\end{aligned}
$$

The part involving $e_{m}^{a}$ describes pure gravity in three dimensions and contains no propagating degrees of freedom, whereas the physical degrees of freedom (the two helicity states of the graviton) reside in the remainder of the Lagrangian. In the equations of motion, one can perform a duality redefinition

$$
\Delta^{2} F_{m n}=\epsilon_{m n p} \partial^{p} B
$$


The integrability of this equation is guaranteed (on-shell) by Maxwell's equation for the vector field $B_{m}$. The resulting equations of motion can be equivalently obtained from the $\sigma$-model Lagrangian

$$
\mathcal{L}=-\frac{1}{4} e R^{(3)}(e)+\frac{1}{8} e g^{m n} \Delta^{-2}\left(\partial_{m} B \partial_{n} B+\partial_{m} \Delta \partial_{n} \Delta\right)
$$

or, in terms of the complex field $Z \equiv B+i \Delta$,

$$
\mathcal{L}=-\frac{1}{4} e R^{(3)}(e)+\frac{1}{2} e g^{m n} \frac{\partial_{m} Z \partial_{n} \bar{Z}}{(Z-\bar{Z})^{2}}
$$

(The superscript (3) will henceforth be dropped.) Note that the field $Z$ is related to the so-called Ernst potential $\mathcal{E}$ via $Z=i \overline{\mathcal{E}}$ (see section 3.2). It is easy to check invariance of (2.1.7) under the $S L(2, \mathbb{R})$ transformations

$$
Z \rightarrow Z^{\prime}=\frac{a Z+b}{c Z+d} ;\left(\begin{array}{ll}
a & b \\
c & d
\end{array}\right) \in S L(2, \mathbb{R})
$$

This is the simplest example of a non-linearly realized symmetry acting on the space of physical field configurations. In the following section, (2.1.7) will be shown to be just a special case of the more general construction.

A more sophisticated example is the reduction of $N=1$ supergravity $[27,28]$ from four to three dimensions. The gravitational sector is, of course, exactly as above, but we now have in addition a four component Majorana vector spinor $\psi_{M}$ (the gravitino), which upon reduction to three dimensions splits into two gravitinos $\psi_{m}^{I}$ and two matter fermions $\chi^{I}$. The extra internal index $I=1,2$ is necessary because spinors in three dimensions have only two components, so every four-dimensional spinor gives rise to two three-dimensional spinors transforming irreducibly under the Lorentz group $S O(1,2)$. In complete analogy with the dreibein in pure gravity the three-dimensional vector spinors $\psi_{m}^{I}$ carry no longer any propagating degrees of freedom, while the two physical degrees of freedom (the helicity $\frac{3}{2}$ states of the gravitino) reside in the "matter fermions" $\chi^{I}$. To work out the reduction with all the necessary field redefinitions is a bit more laborious than in pure gravity (readers interested in the details are invited to have a look at $[15,29]$ where a more complicated example is treated). It is also unnecessary here, for in the following section I will explain the general formalism which will allow us to formulate the models directly in three dimensions by exploiting their symmetry structures.

\subsection{Coset Space $\sigma$-Models and Supergravity}

It can now be shown that the structure of which we just had a first glimpse is actually generic in Kaluza-Klein (super)gravity: there are always hidden symmetries which extend the manifest symmetries obtained in the naive dimensional reduction $[15,16,26]$. One invariably obtains a non-linear $\sigma$-model of the non-compact type based on a coset space $G / H$ with $H$ as the maximally compact subgroup. Of course, the coset space one ends up with depends on which 
theory one starts with, but once the coset has been identified (for instance by simply counting the scalar degrees of freedom), the model is unalterably fixed. This fact can be exploited by taking the three-dimensional Lagrangian as one's point of departure in analyzing the reduction to two dimensions rather than starting from the higher-dimensional theory. In this section, I will first summarize the general formalism for the bosonic $\sigma$-models, which was invented by particle physicists long ago [30] (see also [31] for a more recent treatment) and then present $N=2$ supergravity in three dimensions, which is the locally supersymmetric extension of (2.1.6). The limitation to the $N=2$ theory is mainly for pedagogical reasons, as this is the simplest supersymmetric model to be studied in the present context. However, the notational conventions are such that the comparison with the results described in the appendix is quite straightforward.

To formulate the bosonic $\sigma$-models in a general way [31], consider a maximally symmetric space $G / H$ with the associated Lie algebra decomposition

$$
\mathbf{G}=\mathbf{H} \oplus \mathbf{K}
$$

For the sake of simplicity, we will only consider the case of interest here, where $G$ is non-compact and $H$ is its maximally compact subgroup. The maximal symmetry of the coset space is reflected in the Lie algebra relations

$$
[\mathbf{H}, \mathbf{H}] \subset \mathbf{H} \quad[\mathbf{H}, \mathbf{K}] \subset \mathbf{K} \quad[\mathbf{K}, \mathbf{K}] \subset \mathbf{H}
$$

This decomposition is invariant under the symmetric space automorphism

$$
\tau(\mathbf{H})=\mathbf{H} \quad \tau(\mathbf{K})=-\mathbf{K}
$$

which can alternatively be formulated in terms of Lie group elements $g$ directly through

$$
\tau(g)=\eta^{-1}\left(g^{T}\right)^{-1} \eta
$$

where the matrix $\eta$ depends on the group $G$ (e.g. $\eta=1$ for $G=S L(n, \mathbb{R})$ ).

As already pointed out, our principal example will be the $S L(2, \mathbb{R}) / S O(2)$ coset space. In this case, there are the three generators

$$
Y^{1}=\left(\begin{array}{cc}
1 & 0 \\
0 & -1
\end{array}\right), Y^{2}=\left(\begin{array}{ll}
0 & 1 \\
1 & 0
\end{array}\right), Y^{3}=\left(\begin{array}{cc}
0 & 1 \\
-1 & 0
\end{array}\right)
$$

Clearly,

$$
\operatorname{Tr}\left(Y^{1}\right)^{2}=\operatorname{Tr}\left(Y^{2}\right)^{2}=-\operatorname{Tr}\left(Y^{3}\right)^{2}=2
$$

so $Y^{1}$ and $Y^{2}$ are the non-compact generators, while $Y^{3}$ generates the $S O(2)$ subgroup. I will label the coset generators with capital letters $A, B, \ldots=1,2$.

The scalar fields $\mathcal{V}(x)$ of the associated non-linear $\sigma$ model are now taken to be elements of the group $G$ for all $x$. To reduce the number of physical degrees of freedom to the desired number, one requires invariance under the transformations

$$
\mathcal{V}(x) \rightarrow \mathcal{V}^{\prime}(x)=g^{-1} \mathcal{V}(x) h(x)
$$


so the local $H$ invariance can be used to remove the unwanted degrees of freedom. For instance, we can pick a "unitary" gauge such that

$$
\mathcal{V}(x)=\exp \varphi_{0}(x) \quad \varphi_{0}(x) \in \mathbf{K}
$$

which shows that there are precisely $\operatorname{dim} \mathbf{K}$ physical degrees of freedom. If one acts on this matrix with an arbitrary element $g \in G$, the "unitarity" of the gauge will be lost in general, so a compensating $H$ transformation $h(x ; g, \mathcal{V})$ is necessary to restore the gauge. In this way, the symmetry becomes non-linearly realized on the physical fields $\varphi_{0}(x)$ [30]. Note that if no special gauge is chosen, there are two symmetries acting on $\mathcal{V}$, namely the rigid group $G$ and the local group $H$. Consequently, one can think of the matrix $\mathcal{V}$ as some kind of vielbein, with $G$ and $H$ replacing the groups $G L(D, \mathbb{R})$ and $S O(1, D-1)$, respectively. This analogy will be encountered again in these lectures.

The next step is, of course, the construction of a Lagrangian with the required symmetries. To this aim, consider the Lie-algebra-valued expression

$$
\mathcal{V}^{-1} \partial_{m} \mathcal{V}=Q_{m}+P_{m} \quad Q_{m} \in \mathbf{H}, P_{m} \in \mathbf{K}
$$

or, equivalently,

$$
\mathcal{V}^{-1} D_{m} \mathcal{V} \equiv \mathcal{V}^{-1}\left(\partial_{m} \mathcal{V}-\mathcal{V} Q_{m}\right)=P_{m}
$$

which defines the $H$ covariant derivative $D_{m}$. It is straightforward to verify that $Q_{m}$ transforms indeed as a gauge field with respect to the local group $H$, and that $P_{m}^{\prime}=h^{-1} P_{m} h$. The formula (2.2.7) implies the integrability relations

$$
\begin{aligned}
\partial_{m} Q_{n}-\partial_{n} Q_{m}+\left[Q_{m}, Q_{n}\right] & =-\left[P_{m}, P_{n}\right] \\
D_{m} P_{n}-D_{n} P_{m} & =0
\end{aligned}
$$

The Lagrangian is given by

$$
\mathcal{L}^{\prime}=+\frac{1}{4} e g^{m n} \operatorname{Tr} P_{m} P_{n}=\frac{1}{2} e g^{m n} P_{m}^{A} P_{n}^{A}
$$

where the gravitational fields have been included again. The field equations read

$$
D_{m}\left(\sqrt{g} g^{m n} P_{n}\right)=0
$$

To see that this formulation leads to the same equations as the Einstein Lagrangian of the previous section, take $G=S L(2, \mathbb{R})$ with the matrix $\mathcal{V}$ in the triangular gauge

$$
\mathcal{V}=\left(\begin{array}{cc}
\Delta^{1 / 2} & B \Delta^{-1 / 2} \\
0 & \Delta^{-1 / 2}
\end{array}\right)
$$

Decomposing the expression

$$
\mathcal{V}^{-1} \partial_{m} \mathcal{V}=\left(\begin{array}{cc}
\frac{1}{2} \Delta^{-1} \partial_{m} \Delta & \Delta^{-1} \partial_{m} B \\
0 & -\frac{1}{2} \Delta^{-1} \partial_{m} \Delta
\end{array}\right)
$$

into its components along $\mathbf{K}$ and $\mathbf{H}$, we obtain

$$
P_{m}^{1}=\frac{1}{2} \Delta^{-1} \partial_{m} \Delta, P_{m}^{2}=\frac{1}{2} \Delta^{-1} \partial_{m} B, Q_{m}=\frac{1}{2} \Delta^{-1} \partial_{m} B
$$


Substituting (2.2.15) into (2.2.12), we immediately recover the "matter part" of (2.1.6).

Let us now turn to $N=2$ supergravity. Since the derivation of the results below is standard, I will omit details and only quote the final results, referring the reader to [28] for more information on supergravity technology. In addition to the dreibein $e_{m}^{a}$ and the scalar fields $\Delta$ and $B$ introduced in (2.2.14), the $N=$ 2 theory contains two "matter fermions" $\chi^{I}$ and two gravitinos $\psi_{m}^{I}(I, J, \ldots=$ $1,2)$. In the present formulation, these fields are inert under the action of the rigid group $G$, but do transform under $S O(2)$. There is only one consistent choice of $S O(2)$ charge assignments. For an $S O(2)$ rotation

$$
h=\left(\begin{array}{cc}
\cos \alpha & \sin \alpha \\
-\sin \alpha & \cos \alpha
\end{array}\right)
$$

we must have

$$
\begin{aligned}
P_{m}^{1}+i P_{m}^{2} & \rightarrow e^{2 \imath \alpha}\left(P_{m}^{1}+i P_{m}^{2}\right) \\
\chi^{1}+i \chi^{2} & \rightarrow e^{\frac{3}{2} i \alpha}\left(\chi^{1}+i \chi^{2}\right) \\
\psi_{m}^{1}+i \psi_{m}^{2} & \rightarrow e^{\frac{1}{2} \imath \alpha}\left(\psi_{m}^{1}+i \psi_{m}^{2}\right)
\end{aligned}
$$

This is in accord with our expectation that the "hidden" $S O(2)$ is just the helicity (or transverse) subgroup of the Lorentz group in four dimensions, with $P_{m}^{1} \pm i P_{m}^{2}$ and $\chi^{1} \pm i \chi^{2}$ corresponding to the physical states of helicity \pm 2 and $\pm \frac{3}{2}$, respectively (the supersymmetry transformation parameter $\epsilon^{I}$ transforms like $\psi_{m}^{I}$ ). More general helicity groups are obtained for higher extended theories; for instance, in the maximally extended $N=16$ theory, we have $H=S O(16)$, which contains the helicity group $S O(9)$ of $D=11$ supergravity as a subgroup. Given the charge assignments (2.2.18), we can immediately deduce the form of the fully covariant derivatives acting on the spinors

$$
\begin{aligned}
& \left(D_{m} \chi\right)^{I}=\left(\partial_{m}+\frac{1}{4} \omega_{m a b} \gamma^{a b}\right) \chi^{I}+\frac{3}{2} Q_{m} \epsilon^{I J} \chi^{J} \\
& \left(D_{m} \epsilon\right)^{I}=\left(\partial_{m}+\frac{1}{4} \omega_{m a b} \gamma^{a b}\right) \epsilon^{I}+\frac{1}{2} Q_{m} \epsilon^{I J} \epsilon^{J}
\end{aligned}
$$

which shows how the scalars and the spinors couple via the composite gauge field $Q_{m}$ determined from (2.2.9).

At this point, we could, of course, easily switch to a slightly more economical complex notation, but I prefer not to do so in order to keep the notational uniformity with the $N=16$ results to be discussed in the appendix. Thus we continue to use real fields in the remainder of this subsection, using indices $I, J, \ldots=1,2$ and $A, B, \ldots=1,2$ for the "spinorial" and "vectorial" representations of $S O(2)$, respectively. The full Lagrangian of $N=2$ supergravity is ${ }^{1}$

$1 \quad$ Readers may wonder why there are explicit quartic spinorial terms in this Lagrangian and the transformation rules below, whereas there are no such terms in the usual first (or 1.5) order formalution of $d=4, N=1$ supergravity [27]. The reason for their appearance here is that we are using $\mathbf{1 . 5}$ order formalism only for the three-dimensional spin connection, whereas second order formalism is used for the $S O(2)$ part of the connection, and that one of the components of the vierbein has been dualized. 


$$
\begin{aligned}
\mathcal{L}= & -\frac{1}{4} e R(e)+\frac{1}{2} \epsilon^{m n p} \bar{\psi}_{m}^{I} D_{n} \psi_{p}^{I}+\frac{1}{2} e g^{m n} P_{m}^{A} P_{n}^{A}-\frac{i}{2} e \bar{\chi}^{I} \gamma^{m}\left(D_{m} \chi\right)^{I}- \\
& -e t_{I J}^{A} \bar{\chi}^{I} \gamma^{m} \gamma^{n} \psi_{m}^{J} P_{n}^{A}+\frac{1}{8} e\left(\bar{\chi}^{I} \chi^{I}\right)^{2}-\frac{1}{6} e\left(\bar{\chi}^{I} \gamma^{a} \chi^{J}\right)^{2}+ \\
& +\frac{1}{4} e \bar{\chi}^{I} \gamma_{p} \chi^{J}\left(\bar{\psi}_{m}^{I} \gamma^{m n p} \psi_{n}^{J}-\bar{\psi}_{m}^{I} \gamma^{p} \psi^{m J}\right)-\frac{1}{8} e \bar{\chi}^{I} \chi^{I} \bar{\psi}_{m}^{J} \gamma^{n} \gamma^{m} \psi_{n}^{J}
\end{aligned}
$$

with the "Clebsch Gordan coefficients" $t_{I J}^{A} \equiv \frac{1}{\sqrt{2}}\left(Y^{A}\right)_{I J}$ (see (2.2.5)). It is invariant under the local supersymmetry variations

$$
\begin{gathered}
\delta V_{m}^{a}=i \bar{\epsilon}^{I} \gamma^{a} \psi_{m}^{I} \\
\delta \psi_{m}^{I}=D_{m} \epsilon^{I}+\frac{i}{2} \gamma^{n} \epsilon^{J} \bar{\chi}^{I} \gamma_{m n} \chi^{J} \\
\delta \chi^{I}=i \gamma^{m} \epsilon^{J} \widehat{P}_{m} t_{I J}^{A} \\
\mathcal{V}^{-1} \delta \mathcal{V}=t_{I J}^{A} \bar{\epsilon}^{I} \chi^{J}
\end{gathered}
$$

where $\widehat{P}_{m}^{A}$ denotes the supercovariant extension of $P_{\mu}^{A}$

$$
\widehat{P}_{m}^{A}=P_{m}^{A}-t_{I J}^{A} \bar{\psi}_{m}^{I} \chi^{J}
$$

Later, we will also need the equations of motion for the physical fields, which read

$$
D^{m}\left(P_{m}^{A}-t_{I J}^{A} \bar{\chi}^{I} \gamma^{n} \gamma_{m} \psi_{n}^{J}\right)=\epsilon^{A B} P_{m}^{B}\left(\frac{3}{2} i \epsilon^{I J} \bar{\chi}^{I} \gamma^{m} \chi^{J}+\frac{1}{2} \epsilon^{m n p} \epsilon^{I J} \bar{\psi}_{n}^{I} \psi_{p}^{J}\right)
$$

and

$$
-i \gamma^{m} D_{m}(\widehat{\omega}) \chi^{I}=t_{I J}^{A} \gamma^{n} \gamma^{m} \psi_{n}^{J} \widehat{P}_{m}^{A}+\frac{1}{2}\left(\bar{\chi}^{J} \chi^{J}\right) \chi^{I}
$$

where the Fierz-identity $\left(\bar{\chi}^{I} \gamma^{a} \chi^{J}\right)^{2}=\frac{3}{2}\left(\bar{\chi}^{I} \chi^{I}\right)^{2}$ has been used, and $\widehat{\omega}$ is the supercovariant spin connection

$$
\widehat{\omega}_{m a b}(e, \psi, \chi)=\omega_{m a b}(e)+i \bar{\psi}_{[a}^{I} \gamma_{b]} \psi_{m}^{I}+\frac{i}{2} \bar{\psi}_{a}^{I} \gamma_{m} \psi_{b}^{I}+\frac{1}{4} \epsilon_{m a b} \bar{\chi}^{I} \chi^{I}
$$

The Lagrangian (2.20) is, of course, also invariant under general coordinate transformations in three dimensions, the local Lorentz group $S O(1,2) \cong$ $S L(2, \mathbb{R})$ and $S O(2)$ gauge transformations. It has already been pointed out that the fermions are inert under the rigid group $G$ unless a $S O(2)$ gauge is chosen. If, on the other hand, a gauge is fixed as in (2.2.8), the fermions do transform under $G$ via the induced compensating $H$ rotation $h(x, g ; \mathcal{V})$.

\subsection{Remarks on the Canonical Formalism}

It is well known that the invariance of a Lagrangian under a set of rigid symmetry transformations leads to a conserved charge $\mathcal{Q}$. For the bosonic $\sigma$-models discussed in the preceding section the conserved charge associated with the rigid symmetry under $G$ can be easily obtained by integrating the time component of the conserved current

$$
\mathcal{J}^{m}=\mathcal{V} P^{m} \mathcal{V}^{-1}
$$


over a space-like hypersurface. Note that the vielbein $\mathcal{V}$ converts $P_{m}$, which transforms under $H$ and is inert under the rigid symmetry $G$, into a quantity which is inert under the local symmetry and transforms covariantly under $G$ (cf. $(2.2 .7)$ )

$$
\mathcal{J}^{m} \rightarrow g^{-1} \mathcal{J}^{m} g
$$

The conserved current exists, of course, also for the locally supersymmetric theory. For instance, the conserved $S L(2, \mathbb{R})$ current for $N=2$ supergravity is given by

$$
\begin{aligned}
\mathcal{J}^{m} & =\mathcal{V} Y^{A} \mathcal{V}^{-1}\left(P^{m A}-\bar{\psi}_{n}^{I} \gamma^{m} \gamma^{n} \chi^{J} t_{I J}^{A}\right)+ \\
& +\mathcal{V} Y^{3} \mathcal{V}^{-1}\left(\frac{1}{4} e^{-1} \epsilon^{m n p} \epsilon^{I J} \bar{\psi}_{n}^{I} \psi_{p}^{J}-\frac{3}{4} i \epsilon^{I J} \bar{\chi}^{I} \gamma^{m} \chi^{J}\right)
\end{aligned}
$$

The algebra of symmetry transformations generated by the conserved charges and the interplay between rigid and local symmetries is most conveniently analyzed in the canonical framework. Of course, there is no room here to explain the the basics of canonical gravity in all but the scantiest detail. Fortunately, there are several excellent introductory articles available (see e.g. Isham's contribution in this volume or [32]), to which we can refer the reader for more details. At this point, I would also like to alert the reader to [33] where the issues raised here are discussed in much more detail for maximally extended supergravity in the framework of Ashtekar's formalism.

Canonical gravity is substantially different from other canonical theories in that the Hamiltonian becomes a constraint which, in the classical theory, singles out a physical submanifold in the phase space of general relativity [34]. This constraint is a consequence of the invariance of Einstein's theory under reparametrizations of the time coordinate. In the quantum theory, the constraint must be imposed as an operator constraint on the Hilbert space of wave functionals, and then becomes the famous Wheeler-DeWitt operator $\mathcal{H}_{0}$ [35]. In addition to $\mathcal{H}_{0}$, there are the constraints $\mathcal{H}_{\mathfrak{r}}$ associated with the invariance under spatial diffeomorphisms, and, for the kind of theories we are concerned with here, the constraints associated with the invariance under $H$ gauge transformations, which carry no dynamical content, however. In a locally supersymmetric theory, there is also a supersymmetry constraint $\mathcal{S}$, which generates all the other constraints upon commutation and thus carries both dynamical and kinematic information. Because $\{\mathcal{S}, \mathcal{S}\}=\mathcal{H}_{0}+\ldots$, the equation $\mathcal{S} \Psi=0$ can be regarded as the square root of the Wheeler-DeWitt equation in the same way as the Dirac equation is the square root of the Klein Gordon equation.

A central (and unsolved) problem in canonical gravity is the construction of genuine observables. An observable in the sense of Dirac is any functional $\mathcal{O}$ which does not vanish on the physical subspace generated by the canonical constraints and which weakly commutes with all the constraints. Thus, one demands

$$
\{\phi, \mathcal{O}\} \approx 0
$$


where $\phi$ collectively denotes all the constraint generators. Since, in contrast to ordinary canonical theories, there is no a priori "time" in canonical gravity, (2.3.4) replaces the statement of conservation. The difficult part here is, of course, to get $\mathcal{O}$ to commute with the Hamiltonian constraint, whereas the kinematic constraints are comparatively easy to satisfy. We now see that conserved charges of the above type are good candidates for observables. All one has to show is that $\mathcal{O}=\mathcal{Q}$ weakly commutes with all the constraints according to (2.3.4). In the case at hand, it is rather easy to check that (2.3.4) is indeed satisfied for the charge $\mathcal{Q}$ obtained from (2.3.1). This shows that, in the presence of matter, it is not at all difficult to construct genuine observables, if the matter sector has some rigid invariance group. There is, however, one difference between symmetries introduced "by hand" and the hidden symmetries discussed here: the latter originate from gravity itself. In other words, even in pure gravity, it is possible to construct observables provided one reduces the theory by one (or more) dimensions. ${ }^{2}$

In principle, these statements carry over to the quantum theory. Of course, ordering problems and ill-defined operator products will vitiate many arguments, but let us assume for the moment that these difficulties can be overcome (this is one motivation for considering extended supergravity rather than pure gravity!). Then equation (2.3.4) schematically translates into

$$
[\phi, \mathcal{Q}]=f(\ldots) \phi
$$

where $f(\ldots)$ denotes a functional of the fields. This means that from any given solution $\Psi$ of the Wheeler-DeWitt equation, one can generate another according to $\Psi^{\prime}=\mathcal{Q} \Psi[38,33]$. Consequently, the solutions of the Wheeler-DeWitt equation form multiplets of the rigid symmetry group $G$ (which are, in fact, infinite-dimensional owing to the non-compactness of $G$, if the representation is unitary). Although there is so far no concrete calculation exemplifying this idea, it is clear that what we have here is nothing but the quantum analog of the solution-generating procedures for the classical field equations. The argument also shows that the widely acclaimed uniqueness of the wave function of the universe is merely a consequence of overly restrictive assumptions about the boundary conditions imposed on $\Psi$. In any physically "reasonable" theory, and certainly in any theory originating from higher dimensions, there will be hidden symmetries leading to conserved charges, and the uniqueness of the wave function $\Psi$ will be lost. It is amusing to speculate about the cosmological interpretation of the conserved quantum numbers.

Ultimately, we are interested in the reduction to two dimensions. There now remain only two constraints from the gravitational sector, which when written in the form

2 This was independently noted in [36], where Ashtekar's version of $d=4$ gravity [37] is directly reduced to $d=2$. Their $S L(2, \mathbb{R})$ group is, however, not the same as the one generated by the charge $\mathcal{Q}$ and corresponds to the "Matzner-Misner $S L(2, \mathbb{R})$ " rather than the "Ehlers $S L(2, \mathbb{R})$ " generated by $\mathcal{Q}$ above. It is also not obvious to me whether the loop group identified in [36] is related to the Geroch group, as the latter arises through the interplay of the Ehlers and the Matzner-Misner groups. 


$$
T_{ \pm \pm}=\mathcal{H}_{0} \pm \mathcal{H}_{1} \approx 0
$$

are easily recognizable as the Virasoro constraints well known from string theory [39]. (For chiral reductions to one dimension of the type considered in the appendix, only one of the constraints (2.3.6) survives.) Despite the obvious similarities with string theory, there are also some differences. A glance ahead at (3.2.7) shows that, in contrast to (critical) string theory, the properly defined energy momentum tensor $T_{ \pm \pm}$receives a contribution not only from the matter degrees of freedom, but also from the conformal factor, and that the role of the unphysical target space coordinates $X^{ \pm}$in string theory is now played by certain privileged components of the dreibein ${ }^{3}$. The Wheeler-DeWitt equation then amounts to the familiar requirement that the physical states should be annihilated by $T_{ \pm \pm}$. The really new feature here is the presence of an infinitedimensional rigid symmetry $G^{\infty}$ (to be described in chapter 4), which acts non-trivially on the space of physical states and has no analog in string theory. To what extent then can we generalize the above considerations to this case? Clearly, a first desideratum is the construction of a suitable conserved Kac Moody current $\widehat{\mathcal{J}}_{ \pm}$corresponding to (2.3.1) and (2.3.3). A candidate will be presented in section 3.5. The conserved charges constructed from it are, however, non-local, and the proper canonical treatment has met with some technical difficulties already in much simpler models $[40,41]$. Nonetheless, it is possible to show that $\left\{T_{ \pm \pm}, \mathcal{Q}\right\} \approx 0$, at least for classical theory, although the full algebra of conserved charges remains to be evaluated.

\section{From $d=3$ to $d=2$}

It is the main purpose of this chapter to explain why Einstein's theory as well as more general (supersymmetric) models become integrable after the reduction to two dimensions. At the heart of the construction is the linear system (3.3.2) and its generalizations. The emphasis is more on the practical side in the following sections, and I will give an explicit example illustrating how the method works for colliding plane wave solutions. The supersymmetric case is discussed in section 3.5 .

\subsection{General Remarks}

To reduce the theory to two dimensions, we can choose to drop the dependence on either the time-like coordinate $x^{0}$ or on the space-like coordinate $x^{2}$. In more technical terms, the corresponding solutions will then have one time-like and one space-like or two space-like Killing vectors, respectively. The so-called stationary axisymmetric solutions belong to the first class of solutions; wellknown examples are the Schwarzschild and Kerr-Newman solutions. Of course,

3 See the appendix for a further discussion, if you find these remarks too cryptic. 
many solutions of the stationary axisymmetric type are known [1], but not all of them are physical (as they possess either naked singularities or need stiff "rods" for stability). The second class of solutions describes colliding plane waves. The first non-trivial example of such a solution was discovered in 1970, and more complicated solutions have been found since $[11,12]$. The two cases require a very different treatment: the study of stationary axisymmetric solutions leads to an elliptic boundary value problem while, in the second case, one has to analyze a hyperbolic partial differential equation. The structure of solutions is also very different; for instance, in the stationary axisymmetric case, the singularities are located "on the axis", whereas in the second case, they occur "after the collision".

While the solution generating procedures have been extensively applied to the stationary axisymmetric case, it is only recently that the attention has shifted to the study of colliding plane wave solutions in this framework [13]. It is for this reason, that I will concentrate here on the plane wave case. A new result presented in these lectures is the reconstruction of some recently found colliding plane wave solution via the group theoretical method. These solutions are usually constructed piecewise, by first solving the equations in the interaction region where the collision takes place. Once a solution has been found it is often straightforward to extend it from the lower boundary of the interaction region into two separated regions with pure radiation; in between the incoming waves the space-time is Minkowski (the known solutions can, however, not be continued into the future as they exhibit singularities on the upper boundary of the interaction region, see the figure). It is noteworthy that the group theoretical method yields the pertinent information (i.e. the solution) where it is most interesting, namely in the interaction region.

\subsection{Once More: Einstein Gravity}

As already indicated in the foregoing section, we will take the three-dimensional $\sigma$ models as our point of departure, starting with the Lagrangian (2.2.12) coupled to gravity (or the corresponding supergravity Lagrangian, see section 3.5), rather than reducing the theory directly from $d=4$ to $d=2$. Thus we proceed from

$$
\mathcal{L}=-\frac{1}{4} e R(e)+\frac{1}{4} e g^{m n} \operatorname{Tr} P_{m} P_{n}
$$

As in section 2.1, we can make use of the local Lorentz group to simplify the dreibein and bring it into the form

$$
e_{m}^{a}=\left(\begin{array}{cc}
\lambda \delta_{\mu}^{\alpha} & \rho B_{\mu} \\
0 & \rho
\end{array}\right)
$$

where Greek indices refer to two dimensions. In contrast to the vector $B_{m}$ in (2.1.2), the two-dimensional Kaluza Klein vector $B_{\mu}$ carries no physical degrees of freedom any more, but is an auxiliary field which can be dropped with impunity here (however, it gives rise to higher order fermionic terms in supergravity, see section 3.5 ). So, effectively, 


$$
e_{m}^{a}=\left(\begin{array}{cc}
\lambda \delta_{\mu}^{\alpha} & 0 \\
0 & \rho
\end{array}\right)
$$

from which we can immediately compute the components of the three-metric and other quantities of interest. At this point, we can drop the distinction between flat and curved indices $\alpha, \ldots$ and $\mu, \ldots$ Furthermore, derivatives such as $D_{\mu}$ in (3.2.5) below are only covariantized with respect to $H$, whereas the Lorentz covariantizations have been written out and are properly accounted for by the extra factors of $\rho$ (and the rescalings of fermions by a factor $\lambda^{\frac{1}{2}}$ in supergravity) in all equations referring to two dimensions. It is well known that the form of this gauge is preserved under the conformal reparametrizations

$$
\delta x^{+}=\xi_{-}\left(x^{+}\right) \quad \delta x^{-}=\xi_{+}\left(x^{-}\right)
$$

with the light-cone coordinates $x^{ \pm} \equiv \frac{1}{\sqrt{2}}\left(x^{0} \pm x^{1}\right)$. Substituting the gauge (3.2.3) into the scalar field equation (2.2.13), we arrive at

$$
\rho^{-1} D^{\mu}\left(\rho P_{\mu}\right)=0
$$

Readers should recall that $D_{\mu}$ is the $H$-covariant derivative. The dependence of this equation on $\rho$ is all that remains of three-dimensional gravity.

The remaining equations, which follow from higher dimensions, are the equations for the field $\rho$ and for the conformal factor $\lambda . \rho$ is a free field in two dimensions which can be solved for in terms of two arbitrary functions (left-movers and right-movers)

$$
\rho(x)=\rho_{+}\left(x^{+}\right)+\rho_{-}\left(x^{-}\right)
$$

In the Euclidean case, where the fields depend on two space-like coordinates $\left(x^{1}, x^{2}\right)$ or on the complex coordinates $(z, \bar{z})$ where $z=\frac{1}{\sqrt{2}}\left(x^{1}+i x^{2}\right), \rho$ would be the sum of a holomorphic and an anti-holomorphic part. There remains some freedom in the choice of these functions as we still have the conformal transformations (3.2.4) at our disposal ${ }^{4}$. In the Euclidean case, we can make use of them to identify $\rho$ with one of the coordinates (so-called "Weyl canonical coordinates", see $[1,10]$. For plane-wave type solutions, another choice is usually preferred, see section 3.4 .

The equation of motion for the conformal factor reads (for later convenience, I employ light-cone notation)

$$
\rho^{-1} \partial_{ \pm} \rho \lambda^{-1} \partial_{ \pm} \lambda=P_{ \pm}^{A} P_{ \pm}^{A}+\frac{1}{2} \rho^{-1} \partial_{ \pm}^{2} \rho
$$

Note that the second term on the right hand side can be absorbed into $\lambda$ through the replacement $\lambda \rightarrow \lambda\left(\partial_{+} \rho\right)^{-\frac{1}{2}}\left(\partial_{-} \rho\right)^{-\frac{1}{2}}$, and that this equation determines $\lambda$ only up to a constant factor (this corresponds to the freedom in the

4 Similarities with string theory [39] were already mentioned before. The field $\rho$ corresponds to the target space coordinate $X^{+}$, which can be identified with one of the world-sheet coordinates by a conformal coordinate transformation of the type (3.2.4). The conformal factor $\lambda$, on the other hand, resembles the string coordinate $X^{-}$in that both are dependent fields which can be solved for in terms of the physical fields (the transverse degrees of freedom) by means of the Virasoro constraints. 
normalization of the first term in (3.2.9) below). Observe also that this equation has no analog in flat space theories and this, together with the presence of the field $\rho$ makes an enormous difference. For instance, we cannot simply put $\rho=$ const, as this would imply the vanishing of the right hand side of (3.2.7), which by the positivity of the Killing metric on the subalgebra $\mathbf{K}$ would imply $P_{ \pm}=0$ and leave us only with the trivial solution $\mathcal{V}=$ const (modulo $H$ gauge transformations). It is also evident from (3.2.7) that the theory would be conformally invariant without the extra field $\rho$, as the conformal factor $\lambda$ would then completely decouple from the rest of the theory.

It is instructive to work out the equation of motion (3.2.5) explicitly for the $G / H=S L(2, \mathbb{R}) / S O(2)$ coset space. To do so, we again employ the triangular gauge (2.2.14) together with the explicit expressions for $P_{\mu}$ and $Q_{\mu}$ in (2.2.16). After a little algebra one arrives at the result

$$
\Delta \partial_{\mu}\left(\rho \partial^{\mu} \mathcal{E}\right)=\rho \partial_{\mu} \mathcal{E} \partial^{\mu} \mathcal{E}
$$

in terms of the complex potential $\mathcal{E} \equiv \Delta+i B$. This is just the well known Ernst equation [42]. Solving Einstein's equations is now simply a matter of choosing the appropriate $\rho(x)$, finding a solution to the non-linear partial differential equation (3.2.8) and finally determining the conformal factor $\lambda$ by integration of (3.2.7). For the colliding plane wave solutions, one distinguishes waves with collinear polarization, where $B=0$, or, equivalently, $g_{23}=0$, and waves with non-collinear polarization. For collinearly polarized waves, the non-linear Ernst equation can be reduced to a linear partial differential equation through the replacement $\Delta=\exp \psi$ [43]. For later reference, let us also quote the expression for the four-dimensional line element in the case of collinearly polarized waves, which reads

$$
d s^{2}=2 \Delta^{-1} \lambda d x^{+} d x^{-}-\Delta^{-1} \rho^{2}\left(d x^{2}\right)^{2}-\Delta\left(d x^{3}\right)^{2}
$$

where $\Delta, \rho$ and $\lambda$ depend only on $x^{+}$and $x^{-}$.

\subsection{The Linear System}

The integrability of the non-linear equation of motion (3.2.5) is reflected in the existence of a linear system (or Lax pair). This means that there is a set of linear differential equations, whose compatibility conditions yield just the nonlinear equations that one tries to solve. Such linear systems are well known in flat space integrable models (such as the KdV system or the KP hierarchy), so let us first recall how this method works for flat space $\sigma$-models and ignore the conformal factor $\lambda$, putting $\rho=$ const. To formulate the linear system one must introduce a so-called spectral parameter $t$ as an extra variable and replace $\mathcal{V}(x)$ by a matrix $\widehat{\mathcal{V}}$ which also depends on $t$ [44] (For the reduction to a Euclidean space, one must replace $t \rightarrow i t$, cf. [10])

$$
\mathcal{V}\left(x^{0}, x^{1}\right) \rightarrow \widehat{\mathcal{V}}\left(x^{0}, x^{1} ; t\right)
$$


The linear system is now given by

$$
\widehat{\mathcal{V}}^{-1} \partial_{\mu} \widehat{\mathcal{V}}=Q_{\mu}+\frac{1+t^{2}}{1-t^{2}} P_{\mu}+\frac{2 t}{1-t^{2}} \epsilon_{\mu \nu} P^{\nu}
$$

To prove this assertion, one must check the integrability condition

$$
\partial_{\mu}\left(\hat{\mathcal{V}}^{-1} \partial_{\nu} \hat{\mathcal{V}}\right)-\partial_{\nu}\left(\hat{\mathcal{V}}^{-1} \partial_{\mu} \hat{\mathcal{V}}\right)+\left[\widehat{\mathcal{V}}^{-1} \partial_{\mu} \hat{\mathcal{V}}, \hat{\mathcal{V}}^{-1} \partial_{\nu} \hat{\mathcal{V}}\right]=0
$$

It is an elementary exercise to verify that (3.3.3) is equivalent to the integrability relations (2.2.11) and the scalar equation of motion (3.2.5) with $\rho=1$ (Note that Pohlmeyer's original linear system involves the matrix $\left.\mathcal{U} \equiv \widehat{\mathcal{V}} \mathcal{V}^{-1}\right)$. Once this equation is solved, one can retrieve the original matrix $\mathcal{V}$ by setting $t=0$, i.e. $\mathcal{V}=\widehat{\mathcal{V}}(t=0)$; the equations of motion will then be satisfied automatically. In practice, it is, of course, not so easy to solve (3.3.3), and the solutions of the flat space $\sigma$-models have not been completely classified despite the existence of the linear system [45].

Restoring the $\rho$-dependence in (3.2.5), we see that a constant spectral parameter will no longer do, and that some modification is necessary. The trick is to make the spectral parameter $x$-dependent $[7,8,10]$. It takes a little more work to check that the proper $\rho$-dependence is recovered if $t$ obeys the differential equation $^{5}$

$$
\partial_{\mu} t=-\frac{1}{2} \epsilon_{\mu \nu} \partial^{\nu}\left(\rho\left(t+\frac{1}{t}\right)\right)
$$

This first order equation can be explicitly solved with the result

$$
t(x ; w)=\frac{\sqrt{w+\rho_{+}\left(x^{+}\right)}-\sqrt{w-\rho_{-}\left(x^{-}\right)}}{\sqrt{w+\rho_{+}\left(x^{+}\right)}+\sqrt{w-\rho_{-}\left(x^{-}\right)}}
$$

where $w$ is an integration constant. An equivalent expression is

$$
\frac{1-t}{1+t}=\sqrt{\frac{w-\rho_{-}\left(x^{-}\right)}{w+\rho_{+}\left(x^{+}\right)}}
$$

For fixed $x$, the function $t(x ; w)$ lives on a two-sheeted Riemann surface over the complex $w$-plane, with an $x$-dependent cut extending from $\rho_{-}\left(x^{-}\right)$to $\rho_{+}\left(x^{+}\right)$. The integration constant $w$ can be regarded as an alternative spectral parameter. Evidently, the linear system could also be formulated in terms of $w$, but at the expense of introducing $x$-dependent poles and cuts in the linear system. This phenomenon appears to have no analog for flat space integrable models.

5 A useful alternative version of this formula is

$$
t^{-1} \partial_{+} t=\rho^{-1} \partial_{+} \rho \frac{1-t}{1+t}, t^{-1} \partial_{-} t=\rho^{-1} \partial_{-} \rho \frac{1+t}{1-t}
$$


As we will see in chapter 4 , both $t$ and $w$ play an essential role in the investigation of the underlying group theoretical structure. Below we will also make use of the inverse relation

$$
w=\frac{1}{4} \rho\left(t+\frac{1}{t}\right)-\frac{1}{2}\left(\rho_{+}-\rho_{-}\right)
$$

which shows, incidentally, that the values $t=0$ and $t=\infty$ correspond to $w=\infty$ on the different sheets.

Let us pause to contemplate the significance of the replacement (3.3.1). Obviously, a spectral parameter is required if one wants to enlarge the finite Lie group to its affine extension, and the appearance of $t$ in (3.3.1) fits nicely with this expectation. There is now an infinite hierarchy of fields, as one can see by expanding $\hat{\mathcal{V}}$ in $t$. For convenience, let us go to a generalized "triangular gauge" defined by the requirement that $\widehat{\mathcal{V}}$ should be regular at $t=0[17,10]$, or

$$
\widehat{\mathcal{V}}(x ; t)=\exp \sum_{n=0}^{\infty} t^{n} \varphi_{n}(x)
$$

Substitution into the linear system shows that the higher potentials are all related to the original field $\varphi_{0}$ containing the physical degrees of freedom in a non-linear and non-local fashion; in addition, $\varphi_{0}$ must obey its equation of motion. This is clearly illustrated by the lowest order result

$$
\partial_{\mu} \varphi_{1}=\epsilon_{\mu \nu} \partial^{\nu} \varphi_{0}+\ldots
$$

For the special choice $G / H=S L(2, \mathbb{R}) / S O(2)$ this infinity of new fields in just the infinite hierarchy of "dual potentials" first introduced by Kinnersley and Chitre in their study of the Geroch group [5]. Note also that $t$-dependent transformations will mix the fields in this hierarchy, and therefore constitute an infinite-dimensional generalization of "duality symmetries" of Maxwell's equations in empty space.

Another important feature of the linear system (3.3.2) is its invariance under a generalization of the symmetric space automorphism (2.2.3) [10]. Define (for $\eta=1)$

$$
\tau^{\infty} \widehat{\mathcal{V}}(t) \equiv\left(\widehat{\mathcal{V}}^{T}\right)^{-1}\left(\frac{1}{t}\right)
$$

or, in terms of the Lie algebra,

$$
Q_{\mu} \rightarrow Q_{\mu} \quad P_{\mu} \rightarrow-P_{\mu} \quad t \rightarrow \frac{1}{t}
$$

It is straightforward to verify that

$$
\tau^{\infty}\left(\widehat{\mathcal{V}}^{-1} \partial_{\mu} \widehat{\mathcal{V}}\right)=\hat{\mathcal{V}}^{-1} \partial_{\mu} \widehat{\mathcal{V}}
$$

a property which does not hold for (2.2.9) if we replace $\tau^{\infty}$ by $\tau$. 


\subsection{An Example: Recovering Colliding Plane Wave Solutions}

At this point we can already convince ourselves that the results obtained so far can actually be used to construct non-trivial solutions of Einstein's equation, and I will here discuss a specific example to demonstrate the usefulness of the method. Of central importance for the reconstruction of solutions is the monodromy matrix [10], which is defined as follows.

$$
\mathcal{M}=\widehat{\mathcal{V}} \tau^{\infty} \widehat{\mathcal{V}}^{-1}=\widehat{\mathcal{V}}(x ; t) \widehat{\mathcal{V}}^{T}\left(x ; \frac{1}{t}\right)
$$

A short calculation reveals that

$$
\partial_{\mu} \mathcal{M}=\hat{\mathcal{V}}\left(\hat{\mathcal{V}}^{-1} \partial_{\mu} \hat{\mathcal{V}}-\tau^{\infty}\left(\hat{\mathcal{V}}^{-1} \partial_{\mu} \widehat{\mathcal{V}}\right)\right) \tau^{\infty} \hat{\mathcal{V}}^{-1}=0
$$

where the result (3.3.12) was used. Consequently, $\mathcal{M}$ can only depend on $w$. The solution generating procedure now consists in choosing a matrix $\mathcal{M}(w)$ and finding a factorization as in (3.4.1). In order to be able to extract the components of the metric tensor, the matrix $\widehat{\mathcal{V}}$ should be chosen in a triangular gauge, so that in (3.4.1) one factor is analytic in a neighborhood of $t=0$ while the other is analytic in a neighborhood of $t=\infty$, such that the overlap of the respective domains of analyticity is an annular region. This is a variant of the famous Riemann-Hilbert problem. Observe that the $x$-dependence of $t$, which may have seemed a nuisance in the analysis of the linear system, is absolutely essential here because it is precisely the $x$-dependence of $t$ in the factorization (3.4.1) which generates the $x$-dependence of $\widehat{\mathcal{V}}$ and thereby of $\mathcal{V}$ and the metric. In fact, it is obvious for this reason that this factorization method would not work for a flat space integrable theory.

To facilitate the comparison with the standard literature, I will in this subsection use the notation

$$
u \equiv x^{+} \quad, \quad v \equiv x^{-}
$$

for the light-cone coordinates. Furthermore, the remaining conformal invariance is entirely fixed by choosing the coordinates such that

$$
\rho_{+}(u)=\frac{1}{2}\left(1-2 u^{2}\right), \rho_{-}(v)=\frac{1}{2}\left(1-2 v^{2}\right) \Rightarrow \rho(u, v)=1-u^{2}-v^{2}
$$

The interaction region, where the waves collide, is $0<u, v<1, \rho(u, v)>$ 0 . In the region "before the collision" the solution is obtained by defining $g(u, v) \equiv g(0, v)$ for $-\infty<u \leq 0 ; 0<v<1$ and $g(u, v) \equiv g(u, 0)$ for $-\infty<v \leq 0 ; 0<u<1$. Between the incoming waves, the space-time is Minkowski $(g(u, v)=g(0,0))$. Although the solution is continous across the boundary by construction, the matching introduces certain discontinuities in the higher derivatives (gravitational shock wave). Furthermore, the solution 


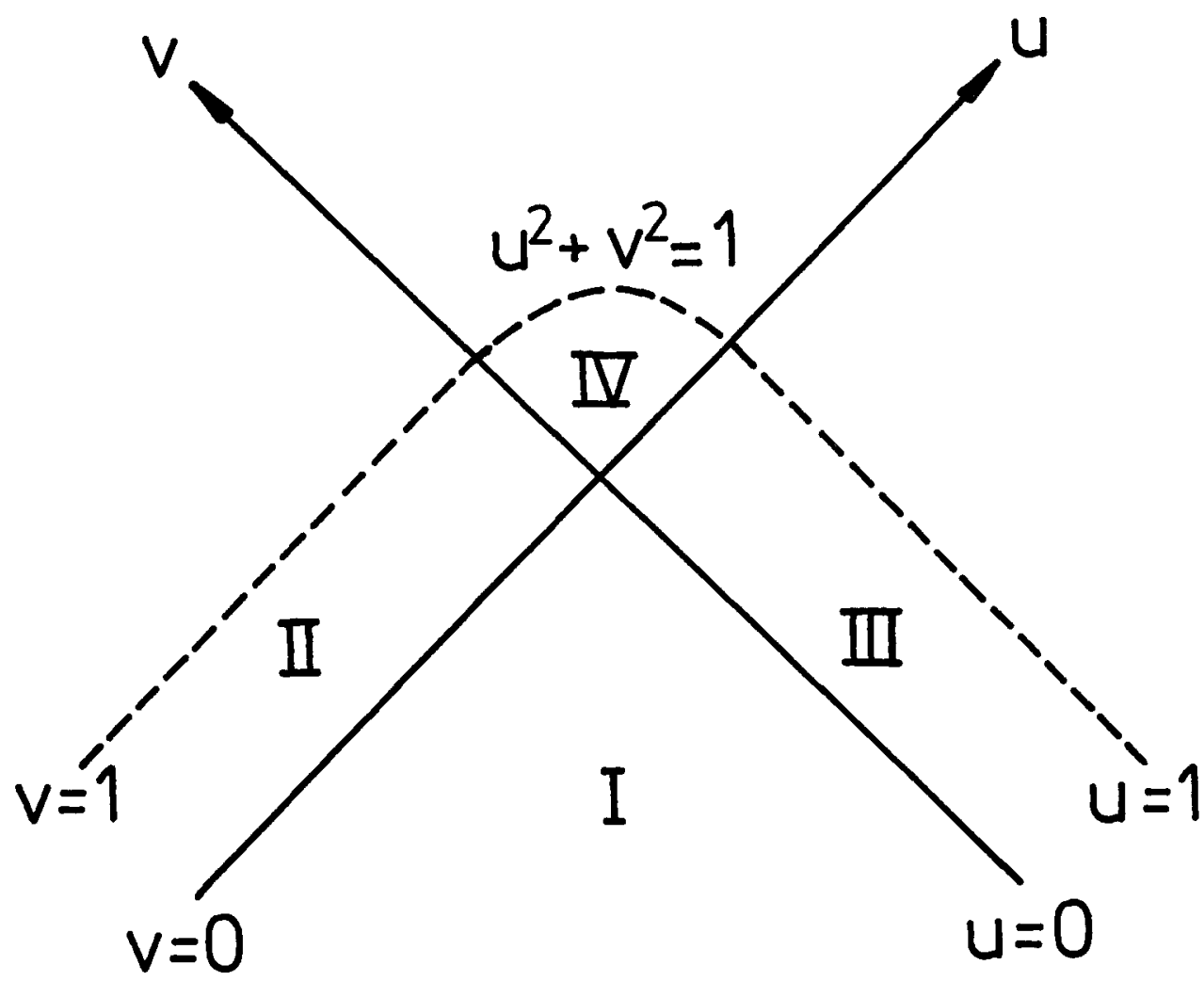

Fig. 1. Space-time for a colliding plane-wave solution. The metric is flat (Minkowski) in (I), and corresponds to pure gravitational radiation in regions (II) and (III). In the interaction region (IV) it is given by the solution (3.4.14) below. The dashed line indicates a curvature (Weyl tensor) singularity [11-13].

cannot be extended beyond $\rho(u, v)=0$ [11-13]. The situation is illustrated in the figure.

We now consider the simplest non-trivial monodromy matrix

$$
\mathcal{M}(w)=\left(\begin{array}{cc}
\frac{w_{0}-w}{w_{0}+w} & 0 \\
0 & \frac{w_{0}+w}{w_{0}-w}
\end{array}\right)
$$

for the special value $w_{0}=\frac{1}{2}$ as an example ${ }^{6}$. Next we use (3.3.7) to derive

$$
w-w_{0}=-\frac{\rho}{4 t_{0}}\left(t-t_{0}\right)\left(\frac{1}{t}-t_{0}\right)
$$

with $t_{0}(u, v) \equiv t\left(u, v ; w=w_{0}\right)$. Substituting (3.4.6) into (3.4.5), and defining the functions (see (3.3.5))

6 For a Euclidean metric, essentially the same monodromy matrix yields the Schwarzschild solution with mass $m=w_{0}$ in Weyl canonical coordinates [18]. The relative minus sign is due to the fact that, for the solution (3.4.9) below, $\widehat{v}(t=0)=\mathcal{V}$ and $\widehat{\mathcal{V}}(t=\infty)=-\tau \mathcal{V}$, so $\mathcal{M}(\infty)=-1$. 


$$
t_{1}(u, v) \equiv t\left(u, v ; w=\frac{1}{2}\right)=\frac{\sqrt{1-u^{2}}-v}{\sqrt{1-u^{2}}+v}>0
$$

and

$$
t_{2}(u, v) \equiv t\left(u, v ; w=-\frac{1}{2}\right)=-\frac{\sqrt{1-v^{2}}+u}{\sqrt{1-v^{2}}-u}<0
$$

(the inequalities hold in the interaction region), we obtain the desired factorization in the form (3.4.1) with

$$
\widehat{\mathcal{V}}(u, v ; t)=\left(\begin{array}{cc}
\sqrt{-\frac{t_{2}}{t_{1}} \frac{t-t_{1}}{t-t_{2}}} & 0 \\
0 & \sqrt{-\frac{t_{1}}{t_{2}} \frac{t-t_{2}}{t-t_{1}}}
\end{array}\right)
$$

Putting $t=0$, we can directly read off the result for $\Delta$ by virtue of $(2.2 .14)$

$$
\Delta=-\frac{t_{1}}{t_{2}}=\frac{1-\xi}{1+\xi}>0
$$

where, on the right hand side, I have introduced the oblate spherical coordinates, widely used in the literature:

$$
\begin{aligned}
& \xi \equiv u \sqrt{1-v^{2}}+v \sqrt{1-u^{2}} \\
& \eta \equiv u \sqrt{1-v^{2}}-v \sqrt{1-u^{2}}
\end{aligned}
$$

To determine the conformal factor, we integrate the equation (3.2.7), using

$$
P_{+}^{1}=\frac{1}{2} \rho^{-1} \partial_{+} \rho\left(\frac{1-t_{1}}{1+t_{1}}-\frac{1-t_{2}}{1+t_{2}}\right)
$$

Some further calculation shows that

$$
\lambda^{2}=8 u v \frac{\left(1-t_{1} t_{2}\right)^{2}}{\left(1-t_{1}^{2}\right)\left(1-t_{2}^{2}\right)}
$$

where the undetermined overall factor has been chosen for convenience. In section 4.2, I will re-derive this result directly from the Breitenlohner-Maison cocycle formula. Collecting all contributions, reexpressing them in terms of the coordinates (3.4.11) and substituting the result into (3.2.9) yields the metric

$$
d s^{2}=(1+\xi)^{2}\left(\frac{d \xi^{2}}{1-\xi^{2}}-\frac{d \eta^{2}}{1-\eta^{2}}\right)-\rho^{2} \frac{1-\xi}{1+\xi}\left(d x^{2}\right)^{2}-\frac{1+\xi}{1-\xi}\left(d x^{3}\right)^{2}
$$

This is just one of the Ferrari Ibanez metrics ${ }^{7}$. It is quite obvious now that more complicated monodromy matrices with off-diagonal terms in (3.4.7) will lead to colliding plane wave solutions with non-collinear polarization of the type discussed in [13].

To conclude this section I will sketch how the factorization works in the general case, given a "reasonable" form of the monodromy matrix (I here follow

7 Equation (2.1) of the first paper in [13] for $n=-1$, to be completely precise. 
the derivation given in [46]). For simplicity, we again assume absence of higher order poles in the monodromy matrix

$$
\mathcal{M}(w)=Y+\sum_{i=1}^{N} \frac{A_{\imath}}{w-w_{i}}
$$

where $Y=\mathcal{M}(\infty)$ and the $A_{i}$ are constant symmetric but otherwise arbitrary matrices. Defining $t_{i} \equiv t\left(x ; w_{i}\right)$, we have

$$
\frac{1}{w-w_{i}}=\nu_{i}\left(\frac{t_{i}}{t-t_{i}}+\frac{1}{1-t_{i} t}\right)
$$

with the functions $\nu_{i}$

$$
\nu_{i}(x) \equiv-4 \rho^{-1} \frac{t_{z}}{1-t_{z}^{2}}
$$

Inspired by the explicit result (3.4.9), we make the following ansatz for $\widehat{\mathcal{V}}$

$$
\widehat{\mathcal{V}}(t)=B_{0}+\sum_{i} \frac{B_{i}}{t-t_{i}}
$$

where the functions $B_{0}(x)$ and $B_{1}(x)$ are to be determined. Once the solution of the factorization problem has been brought into the form (3.4.18), we can immediately read off the result for $\mathcal{V}(x)$, which is

$$
\mathcal{V}=B_{0}-\sum_{i} \frac{B_{i}}{t_{i}}
$$

Inserting the ansatz (3.4.18) into (3.4.1), we obtain the following matrix equations after a little algebra

$$
\begin{aligned}
Y & =B_{0} \mathcal{V}^{T} \\
\nu_{i} t_{2} A_{i} & =B_{i}\left(\mathcal{V}^{T}+\sum_{\jmath} \frac{B_{j}^{T}}{t_{j}} \frac{1}{1-t_{i} t_{j}}\right) \\
\nu_{\imath} t_{\imath} A_{i} & =\left(B_{0}+\sum_{\jmath} B_{j} \frac{t_{i}}{1-t_{\imath} t_{\jmath}}\right) B_{i}^{T}
\end{aligned}
$$

Assuming that these equations can be solved, we have therefore succeeded in reducing the problem of solving a non-linear partial differential equation to a purely algebraic problem in this way! Finally, we have to make sure that acting with a derivative on $\hat{\mathcal{V}}$, we do get the right pole structure for the linear system. After a little further calculation (not forgetting the derivatives on $t$ and $t_{\mathfrak{t}} !$ ), we find 


$$
\begin{aligned}
\partial_{+} \widehat{\mathcal{V}}= & \partial_{+} B_{0}+\sum_{i} \frac{1}{t-t_{i}}\left(\partial_{+} B_{\imath}+\rho^{-1} \partial_{+} \rho B_{i} \frac{t_{i}}{1+t_{i}}\right)- \\
& -\frac{1-t}{1+t} \rho^{-1} \partial_{+} \rho \sum_{i} \frac{1}{t-t_{\imath}} \frac{B_{\imath}}{1+t_{i}}
\end{aligned}
$$

which displays the desired structure explicitly.

\subsection{Extension to Supergravity}

The foregoing considerations can be generalized to supergravity theories [19]. In this section, I will give the results for $N=2$ supergravity, making use of the results in section 2.2, and also present the Kac-Moody generalization of the current (2.3.3), which can be cast into a very simple and model-independent form. As before much labor is saved if we start from the three dimensional Lagrangian (2.2.20) rather than $N=1$ supergravity in four dimensions. Again it proves convenient to fix certain gauges. For the dreibein, we make the same choice as in (3.2.2). In addition, we invoke local supersymmetry (2.2.21) to put

$$
\psi_{a}^{I}=\left(\gamma_{\alpha} \psi^{I}, \psi_{2}^{I}\right)
$$

(the index $a$ is a flat Lorentz index in three dimensions). Thus, in two dimensions, we have the correspondence

$$
\lambda \leftrightarrow \psi^{I} \quad, \quad \rho \leftrightarrow \psi_{2}^{I}
$$

It is important that, just as in the bosonic theory, there is a residual invariance which preserves the form of this gauge. In addition to the conformal reparametrizations (3.2.4), we now have the residual superconformal transformations with a parameter $\epsilon^{I}$ subject to the constraint

$$
\gamma^{\nu} \gamma^{\mu} D_{\nu} \epsilon^{I}=0
$$

This residual invariance can be used to put $\psi_{2}^{I}=0$ in the equations of motion (however, it is also possible to keep the gravitinos by not fixing this remaining gauge, see [20]). A further simplification of the equations of motion of the physical fields occurs when one passes to second order formalism and eliminates the auxiliary vector $B_{\mu}$ in (3.2.2) through its equation of motion [19]. Defining $\epsilon_{\mu \nu} F \equiv \lambda^{-1} \rho\left(\partial_{\mu} B_{\nu}-\partial_{\nu} B_{\mu}\right)$ and rescaling the fermions by a factor $\lambda^{\frac{1}{2}}$, a calculation completely analagous to the one leading to (2.1.4) reveals that the field $F$ appears only in the following combination

$$
\mathcal{L}^{\prime}=\frac{1}{8} \rho F^{2}-\frac{1}{4} \rho F\left(\bar{\psi}^{I} \psi^{I}+\frac{1}{2} \bar{\chi}^{I} \chi^{I}\right)
$$

Elimination of $F$ therefore produces the following extra quartic terms

$$
\mathcal{L}^{\prime}=-\frac{1}{8} \rho\left(\bar{\psi}^{I} \psi^{I}\right)^{2}-\frac{1}{8} \rho \bar{\psi}^{I} \psi^{I} \bar{\chi}^{J} \chi^{J}-\frac{1}{32} \rho\left(\bar{\chi}^{I} \chi^{I}\right)^{2}
$$

to which we must add the terms originating from the second order spin connection 


$$
\mathcal{L}^{\prime \prime}=\frac{1}{8} \rho\left(\bar{\psi}^{I} \psi^{I}\right)^{2}+\frac{1}{8} \rho \bar{\psi}^{I} \psi^{I} \bar{\chi}^{J} \chi^{J}-\frac{3}{32} \rho\left(\bar{\chi}^{I} \chi^{I}\right)^{2}
$$

Adding up these contributions, we see that the remaining gravitino components $\psi^{I}$ decouple, since no further contributions involving $\psi^{I}$ arise from the extra terms in (2.2.23) if we put $\psi_{2}^{I}=0$. Taking into account all the $(\bar{\chi} \chi)^{2}$ terms and rescaling the fermion fields by $\lambda^{\frac{1}{2}}$, we arrive at the equations of motion

$$
\begin{aligned}
& \rho^{-1} D^{\mu}\left(\rho P_{\mu}^{1}\right)=3 i P_{\mu}^{2} \bar{\chi}^{1} \gamma^{\mu} \chi^{2} \\
& \rho^{-1} D^{\mu}\left(\rho P_{\mu}^{2}\right)=-3 i P_{\mu}^{2} \bar{\chi}^{1} \gamma^{\mu} \chi^{2}
\end{aligned}
$$

and

$$
\begin{aligned}
& -i \rho^{-\frac{1}{2}} \gamma^{\mu} D_{\mu}\left(\rho^{\frac{1}{2}} \chi^{1}\right)=\left(\bar{\chi}^{2} \chi^{2}\right) \chi^{1} \\
& -i \rho^{-\frac{1}{2}} \gamma^{\mu} D_{\mu}\left(\rho^{\frac{1}{2}} \chi^{2}\right)=\left(\bar{\chi}^{1} \chi^{1}\right) \chi^{2}
\end{aligned}
$$

These equations constitute the supersymmetric generalization of the Ernst equation (3.2.8).

The bosonic linear system (3.3.2) admits the following generalization for $N=2$ supergravity

$$
\begin{aligned}
\hat{\mathcal{V}}^{-1} \partial_{\mu} \widehat{\mathcal{V}} & =\left(\frac{1+t^{2}}{1-t^{2}} P_{\mu}^{A}+\frac{2 t}{1-t^{2}} \epsilon_{\mu \nu} P^{\nu A}\right) Y^{A} \\
& +\left(Q_{\mu}-\frac{2 t^{2}}{\left(1-t^{2}\right)^{2}} 3 i \bar{\chi}^{1} \gamma_{\mu} \chi^{2}-\frac{t\left(1+t^{2}\right)}{\left(1-t^{2}\right)^{2}} 3 i \bar{\chi}^{1} \gamma^{3} \gamma_{\mu} \chi^{2}\right) Y^{3}
\end{aligned}
$$

To prove this assertion, the integrability relation (3.3.3) must again be checked; the explicit calculations are now considerably more tedious than in the bosonic case due to the extra contributions arising from the derivatives on the spectral parameter and the quartic spinor terms. The main feature distinguishing (3.5.9) from the bosonic result (3.3.2) is the appearance of higher order poles in the spectral parameter $t$ (even higher order poles appear when the gravitinos are retained [20]). For $N=2$, some information is lost in the linear system because the cubic terms on the right hand side of (3.5.8) as well as the coupling between $\chi$ and the gauge field $Q_{\mu}$ drop out because the subgroup $S O(2)$ is abelian. This is no longer true for non-abelian $H$. Apart from this, the on-shell information of $N=2$ supergravity is now entirely contained in the matrix $\widehat{\mathcal{V}}$. It is noteworthy that the linear system with the fermions remains invariant under the generalized symmetric space automorphism $\tau^{\infty}$ of (3.3.12).

There is also an analog of the conserved current (2.3.3) [38]. It is

$$
\begin{aligned}
\widehat{\mathcal{J}}_{\mu}=\rho K \hat{\mathcal{V}} & \left(\left(\frac{1+t^{2}}{1-t^{2}} P_{\mu}^{A}+\frac{2 t}{1-t^{2}} \epsilon_{\mu \nu} P^{\nu A}\right) Y^{A}-\right. \\
& \left.-\left(\frac{1+6 t^{2}+t^{4}}{\left(1-t^{2}\right)^{2}} \frac{3}{2} i \bar{\chi}^{1} \gamma_{\mu} \chi^{2}+\frac{4 t\left(1+t^{2}\right)}{\left(1-t^{2}\right)^{2}} \frac{3}{2} i \bar{\chi}^{1} \gamma^{3} \gamma_{\mu} \chi^{2}\right) Y^{3}\right) \widehat{\mathcal{V}}^{-1}
\end{aligned}
$$

where the factor $K$ satisfies the differential equation 


$$
K^{-1} \partial_{\mu} K=\frac{4}{1-t^{2}} \epsilon_{\mu \nu} \partial^{\nu} t
$$

and is explicitly given by ${ }^{8}$

$$
\rho K=-8 \rho^{-1} \frac{t^{2}}{\left(1-t^{2}\right)^{2}}=\frac{2}{1-t^{2}} \frac{\partial t}{\partial w}
$$

The somewhat unexpected prefactor $\rho K$ ensures that the divergence $\widehat{\mathcal{J}}_{\mu}$ is indeed proportional to the equations of motion (with the correct factors of $\rho$ !) and therefore vanishes. Readers skeptical that we are on the right track with the above expression for the Kac Moody current should note that in flat space (i.e.rigidly) supersymmetric models, one finds exactly the same expression for the conserved current [47], but without the prefactor $\rho K$, since we have $\rho=1$ and $t=$ const in that case.

There is a very nice simplification of the result (3.5.10). To derive it, re-write (3.5.10) as follows

$$
\widehat{\mathcal{J}}_{\mu}=\frac{1}{2} \rho K\left(1-t^{2}\right) \hat{\mathcal{V}}\left(\frac{\partial}{\partial t}\left(\epsilon_{\mu \nu} \hat{\mathcal{V}}^{-1} \partial^{\nu} \widehat{\mathcal{V}}\right)\right) \hat{\mathcal{V}}^{-1}
$$

Now using

$$
\frac{\partial}{\partial t}\left(\hat{\mathcal{V}}^{-1} \partial_{\mu} \widehat{\mathcal{V}}\right)=\partial_{\mu}\left(\hat{\mathcal{V}}^{-1} \frac{\partial \hat{\mathcal{V}}}{\partial t}\right)+\left[\hat{\mathcal{V}}^{-1} \partial_{\mu} \hat{\mathcal{V}}, \hat{\mathcal{V}}^{-1} \frac{\partial \hat{\mathcal{V}}}{\partial t}\right]+\hat{\mathcal{V}}^{-1}\left[\frac{\partial}{\partial t}, \partial_{\mu}\right] \widehat{\mathcal{V}}
$$

and

$$
\left[\frac{\partial}{\partial t}, \partial_{\mu}\right]=-\partial_{\mu}\left(\frac{\partial w}{\partial t}\right) \frac{\partial}{\partial w}
$$

together with (3.5.12) above, some re-arrangement leads to a much more elegant formula for the Kac Moody current

$$
\widehat{\mathcal{J}}_{\mu}=\epsilon_{\mu \nu} \partial^{\nu}\left(\frac{\partial \widehat{\mathcal{V}}}{\partial w} \widehat{\mathcal{V}}^{-1}\right)
$$

In this form the result is completely general and model-independent, and remains valid in particular when gravitinos are included. We will come back to it in section 4.2 when discussing the central term.

Finally, a remark on the status of fermionic solutions is in order. To date, no such solutions of the supersymmetric Ernst equation (3.5.7) and (3.5.8) describing colliding supergravitational plane waves are known, but there is no reason of principle why such solutions should not exist. This is strongly suggested by the existence of fermionic solutions extending the extreme Reissner-Nordstrom solution [48]. There remains, of course, the question of how to interpret Grassmann valued solutions. Their main importance in the present context derives

8 This simple solution was overlooked in [38], where it was erroneously claimed that $K$ is a non-local function of $t$. 
from the new insights one might hope to gain into the structure of the linear system (3.5.9).

\section{Group Theoretical Structure}

As already emphasized in the introduction, one of the main motivations for studying the reduction of gravity and supergravity theories to low dimensions is the search for a better understanding of the underlying symmetry structures. In this section, I will describe the emergence of infinite-dimensional symmetries of the affine Kac-Moody type for the purely bosonic theory. Although the work of the last few years has provided us with a fairly satisfactory understanding in that case, there have been some surprising developments such as the discovery of the central extension [17,10] and of a Virasoro algebra acting on the space of solutions $[49,18]$. These will be reviewed in sections 4.2 and 4.3 , respectively. A new result is the formula (4.2.18) for the variation of the conformal factor. While the first two sections of this chapter deal mostly with the bosonic theory, the last section is devoted to a discussion of the supersymmetric theory, where some perplexing questions remain. These are in part related to the fact that, until now, no fermionic analog of the infinite hierarchy of dual potentials in (3.3.7) has been found: the chiral fermions behave like eigenstates under the duality rotations associated with the infinite-dimensional extensions of $G$ (cf. equation (4.2.29) below). This and our lack of understanding of how to treat fermionic solutions in the present framework may be taken as a hint at the existence of yet more symmetries. In fact, by considering the hyperbolic extension of certain affine Dynkin diagrams, B.Julia was led to conjecture the emergence of hyperbolic symmetries in the reduction to one dimension, and in particular of an $E_{10}$ symmetry in the maximally extended $N=16$ supergravity theory [24]. In section 4.3, I will argue that the appearance of such algebras is related to the introduction of fermionic degrees of freedom. The suggestive relation between hyperbolic Lie algebras and locally supersymmetric theories is most impressively exemplified by the fact that the "maximally extended" simply laced hyperbolic Lie algebras are extensions of $E_{8}$ and $D_{8}=S O(16)$ [23], which are precisely the groups that appear in the maximally extended $N=16$ supergravity theory.

\subsection{Infinite Dimensional Coset Spaces}

In section 2.2 , some pertinent results for finite-dimensional $\sigma$-models were briefly reviewed. We would now like to generalize these results to the infinitedimensional case. The appearance of the spectral parameter $t$ in section 3.3 was the first evidence for the emergence of an affine Kac-Moody symmetry. The situation is somewhat complicated by the $x$-dependence of $t$, and in section 3.3 we also noted the existence of another spectral parameter $w$, which 
does not depend on $x$, and which is, in principle, interchangeable with $t$. As already pointed out before, both these parameters are essential for the generalizations of the various concepts introduced in section 3.3. To see why this is so, recall that the Kac Moody group $G^{\infty}$, which extends (and contains) $G$ must act rigidly. Therefore the elements of this group must depend on $w$ rather than $t$. On the other hand, the infinite-dimensional generalization $\tau^{\infty}$ of the symmetric space automorphism introduced in (3.3.10) involves the parameter $t$, and therefore automatically brings in an $x$-dependence. This shows already. that we must introduce matrices which depend on both $x$ and $w$, if we want to define an infinite-dimensional extension of the coset space $G / H$. We are thus naturally led to consider the matrix $\widehat{\mathcal{V}}(x, t(x, w))$ as an element of $G^{\infty}$ for each (fixed) $x$. The actual definition of $G^{\infty}$ also requires some technical assumptions about the regularity properties of $g(w) \in G^{\infty}$ such as the requirement that $g(w)$ should be a meromorphic function of $w$, but I will not further elaborate on this issue here (see [10] for a discussion). The subgroup $H^{\infty}$ extending $H$ is then defined to consist of all matrices $h(x ; t)$ in $G^{\infty}$ invariant under the generalized automorphism $\tau^{\infty}$, or

$$
h(x ; t) \in H^{\infty} \Longleftrightarrow(h(x ; t))^{-1}=h\left(x ; \frac{1}{t}\right)^{T}
$$

The Lie algebras associated with $G^{\infty}$ and $H^{\infty}$ will be called $\mathbf{G}^{\infty}$ and $\mathbf{H}^{\infty}$. An element of $\mathbf{G}^{\infty}$ is written as

$$
\delta g(w)=\sum_{n=-\infty}^{\infty} \delta g_{n} w^{n}
$$

whereas the general element of $\mathbf{H}^{\infty}$ has the form

$$
\delta h(t)=\delta h_{0}+\sum_{n>0} \delta h_{n}\left(t^{n}+\frac{1}{t^{n}}\right)+\sum_{n>0} \delta k_{n}\left(t^{n}-\frac{1}{t^{n}}\right)
$$

with $\delta h_{n} \in \mathbf{H}$ and $\delta k_{n} \in \mathbf{K}$. So, roughly speaking, $\mathbf{H}^{\infty}$ is "half" of the Lie algebra $\mathbf{G}^{\infty}$.

We are now interested in finding and classifying the solutions $\widehat{\mathcal{V}}(x, t)$ of the linear system (3.3.2). According to (3.3.12), the expression $\widehat{\mathcal{V}}^{-1} \partial_{\mu} \widehat{\mathcal{V}}$ must be an element of $\mathbf{H}^{\infty}$, which, by construction, is "pure gauge". This suggests that ${ }^{9}$.

$$
\widehat{\mathcal{V}}(x, t)=g^{-1}(w) h(x, t)
$$

because we still have the freedom of multiplication by an $x$-independent but $w$-dependent matrix from the left, since $\widehat{\mathcal{V}}$ is an element of $G^{\infty}$, and not just $H^{\infty}$. The explicit $t$-dependence on the right hand side of (3.3.2) imposes a stronger constraint on $\widehat{\mathcal{V}}^{-1} \partial_{\mu} \hat{\mathcal{V}}$, since matrices of the form (4.1.3) will not yield

$9 \quad$ The question of whether indeed all solutions of (3.3.3) can be represented in this way is related to the question of whether the group $G^{\infty}$ acts transitively, see the discussion below. 
the $t$-dependence prescribed by (3.3.2) in general. In addition, we demand that $\widehat{\mathcal{V}}$ should be regular at $t=0$, so we can recover $\mathcal{V}$ in the limit $t \rightarrow 0$. This requirement is tantamount to the existence of a triangular gauge for $\widehat{\mathcal{V}}$ as in (3.3.8). For arbitrary matrices in (4.1.4), $\widehat{\mathcal{V}}$ will, however, fail to satisfy these requirements, because the replacement of $w$ by $t$ through (3.3.7) introduces poles at $t=0$. Nonetheless, we can argue that there exists a special $h_{0}(x, t ; g)$, implicitly depending on $g(w)$, such that

$$
\widehat{\mathcal{V}}_{0}(x, t)=g^{-1}(w) h_{0}(x, t ; g)
$$

has the desired properties. To see this, let us first determine the monodromy matrix from (4.1.4), which is $\mathcal{M}(w)=\left(g^{T}(w) g(w)\right)^{-1}$. If the matrix equations (3.4.20) can be solved, there exists a solution $\widehat{\mathcal{V}}_{0}$ to the Riemann Hilbert factorization problem, which is of the form (3.4.18) and thus indeed regular at the origin. The $H^{\infty}$ matrix in (4.1.5) is then simply $h_{0}=g(w) \widehat{\mathcal{V}}_{0}$. Differentiating this solution generates at most first order poles at $t= \pm 1$ by (3.4.21), and this, in turn, guarantees the absence of higher order poles from the linear system (3.3.2). Altogether, these arguments show that, given a suitably regular matrix $g(w)$ in (4.1.3), we can always construct a solution of the linear system with the requisite properties.

These considerations make it highly plausible that the space of solutions to the non-linear equation (3.2.5) can be identified with the coset space $G^{\infty} / H^{\infty}$. To see this, consider the space of all matrices $\widehat{\mathcal{V}}(x ; t)$ with $\widehat{\mathcal{V}}^{-1} \partial_{\mu} \widehat{\mathcal{V}} \in \mathbf{H}^{\infty}$, but not necessarily of the special form (3.3.2). On this space, there is a combined action of $G^{\infty} \times H^{\infty}$

$$
\widehat{\mathcal{V}}(x ; t) \rightarrow \widehat{\mathcal{V}}^{\prime}(x ; t)=g^{-1}(w) \widehat{\mathcal{V}}(x ; t) h(x ; t)
$$

Thus the group $G^{\infty}$ acts rigidly from the left, while $H^{\infty}$ acts as a gauge group from the right. The infinitesimal counterpart of (4.1.6) is

$$
\delta \widehat{\mathcal{V}}(x ; t)=-\delta g(w) \hat{\mathcal{V}}(x ; t)+\widehat{\mathcal{V}}(x ; t) \delta h(x ; t)
$$

where $\delta g(w)$ and $\delta h(x ; t)$ are to be expanded as in (4.1.2) and (4.1.3), but with constant $\delta g_{n}$ and $x$-dependent $\delta h_{n}$ and $\delta k_{n}$. Observe that through the elevation of $H^{\infty}$ to a gauge group, the infinitely many "dual potentials" introduced in (3.3.8) (now with $n \in \mathbb{Z}$ ) become independent gauge degrees of freedom. The transformation law (4.1.6) generalizes (2.2.7) to the infinite-dimensional case. The coset space $G^{\infty} / H^{\infty}$ can now be defined in complete analogy with the finite-dimensional space $G / H$ : two matrices $\widehat{\mathcal{V}}$ and $\hat{\mathcal{V}}^{\prime}$ will be called gauge equivalent if and only if there exists a matrix $h \in H^{\infty}$ such that $\hat{\mathcal{V}}^{\prime}=\widehat{\mathcal{V}} h$. The equivalence classes of such matrices constitute the elements of the coset space $G^{\infty} / H^{\infty}$. By the above reasoning, each equivalence class contains one particular element $\widehat{\mathcal{V}}_{0}$, which is analytic at $t=0$ and from which the matrix $\mathcal{V}$ solving the field equations (3.2.5) can be reconstructed. Therefore, the equivalence classes are in one-to-one correspondence with the solutions of (3.3.2), and thereby of (3.2.5). Conversely, one can proceed from a given solution $\widehat{\mathcal{V}}_{0}$ 
and act on it with an arbitrary matrix $g(w) \in G^{\infty}$. This transformation will in general take us out of the coset to which $\widehat{\mathcal{V}}_{0}$ belongs, and a compensating $H^{\infty}$ rotation must be applied to restore the triangular gauge.

In summary, the affine Kac-Moody group $G^{\infty}$ acts on the space of matrices $\widehat{\mathcal{V}}$ satisfying $\hat{\mathcal{V}}^{-1} \partial_{\mu} \hat{\mathcal{V}} \in \mathbf{H}^{\infty}$. If we insist that $\hat{\mathcal{V}}$ be triangular as in (3.3.8), or, equivalently, that $\hat{\mathcal{V}}^{-1} \partial_{\mu} \hat{\mathcal{V}}$ be subject to the more restrictive condition (3.3.2), the symmetry is realized non-linearly. Since we can then extract the solution of the original non-linear field equation from $\widehat{\mathcal{V}}$, there is also a non-linear action of $G^{\infty}$ on the space of solutions. There is an obvious analogy between $\widehat{\mathcal{V}}$ and the vielbein in general relativity, with $G^{\infty}$ replacing $G L(D, \mathbb{R})$ and $H^{\infty}$ the Lorentz group (however, $G^{\infty}$ and $H^{\infty}$ contain only their transverse subgroups). For this reason, and because $G^{\infty}$ is infinite-dimensional, the matrix $\widehat{\mathcal{V}}$ might be appropriately referred to as " $\infty$-bein". This analogy is deficient only insofar as all the elements of $G^{\infty} / H^{\infty}$ are "on shell" by construction, whereas this is not the case for the finite-dimensional coset spaces of section 2.2 .

To be sure, there remain some thorny technical issues. One is the question of whether all the solutions of (3.2.5) can indeed be obtained in this way, or, equivalently, whether the group $G^{\infty}$ acts transitively on the space of solutions, and all solutions can be "reached" from $\widehat{\mathcal{V}}_{0}=1$ via (4.1.6). For stationary axisymmetric solutions of Einstein's equations, this has been demonstrated modulo some technical assumptions in [50]. For the plane wave solutions discussed in section 3.4, the corresponding result is still lacking ${ }^{10}$. A second, and perhaps more significant indication of our incomplete understanding is the existence of further symmetries. The considerations above did not take into account the conformal factor, a defect that will be remedied in the following section. As a consequence, the space of solutions is actually bigger, and the coset space defined above must be modified. In fact, there are more symmetries at work even in the purely bosonic theory, and in section 4.3, a Virasoro type symmetry on the solution space will be exhibited. There, we will also return to the fermionic theories.

\subsection{The Central Extension}

It is well known that every affine Kac-Moody algebras admits a central extension [23]. In physical examples, the appearance of such a term is usually linked to quantization (see [51] for a very readable introduction). It is therefore rather surprising that, for the affine algebra $\mathbf{G}^{\mathbf{\infty}}$, a central term is already present in the classical theory $[17,10]$. Moreover, this central term is not a $c$-number, but acts as a scaling operator on the conformal factor. In this section, a derivation of this result is given which generalizes previous ones and also applies to supergravity theories. Although the final result (4.2.18) is completely general, the proof is only given for the bosonic model in order to avoid cumbersome formulas. Furthermore, I will employ light-cone notation from now on (with

10 At least to the best of the author's knowledge. 
the usual conventions $V^{ \pm} \equiv \frac{1}{\sqrt{2}}\left(V^{0} \pm V^{1}\right)$ for vectors, etc. $)$, since the derivation becomes much more transparent in this way. Details of the calculation are provided only for positive $(t)$ chirality components, since they are the exactly the same for the opposite chirality.

The linear system (3.3.2) will now be used in the form

$$
\widehat{\mathcal{V}}^{-1} \partial_{+} \widehat{\mathcal{V}}=Q_{+}+\frac{1-t}{1+t} P_{+} \quad, \quad \widehat{\mathcal{V}}^{-1} \partial_{-} \widehat{\mathcal{V}}=Q_{-}+\frac{1+t}{1-t} P_{-}
$$

so the right and left moving components of the linear system have poles at $t=-1$ and $t=1$, respectively. The correspondence between the chirality and the location of the poles in $t$ remains valid for supergravity, as one can easily verify from (3.5.9) and (3.5.10).

The starting point is equation (3.2.7), which determines the conformal factor in terms of the "matter fields" up to an overall factor, and which we now write as

$$
\rho^{-1} \partial_{ \pm} \rho \lambda^{-1} \partial_{ \pm} \lambda=\frac{1}{2} \operatorname{Tr} P_{ \pm} P_{ \pm}
$$

disregarding the term involving second derivatives of $\rho$, which can be absorbed into a redefinition of $\lambda$, from now on. To analyze the action of $G^{\infty}$ on $\lambda$, we must first find out how this group acts on the right hand side of (4.2.2). From (4.1.6) and (3.3.2), it is clear that $P_{ \pm}$and $Q_{ \pm}$are inert under the action of $G^{\infty}$, and therefore this group can act only via the induced compensating $H^{\infty}$ rotation needed to restore the generalized triangular gauge. Hence, we must examine the compensating generalized duality rotations in a little more detail. It is advantageous to analyze infinitesimal transformations of the form (4.1.2) with a "cutoff" on the number of components, i.e. $\delta g_{n}=0$ for $|n|$ sufficiently large. Assuming $\widehat{V}$ to be regular at $t=0$, we now demand that the the compensating $\mathbf{H}^{\infty}$ variation in (4.1.7) should be chosen in such a manner that $\delta \widehat{\mathcal{V}}$ is also regular at $t=0$. Inserting the expansion (4.1.2) into (4.1.7) and using (3.3.7), we see that only the positive powers of $w$ in (4.2.1) lead to poles in $t$ which must be compensated by an appropriate $\delta h(t)$. If, on the other hand, only negative powers of $w$ appear, we can put $\delta h(t)=0$ because $w^{-1}=4 \rho^{-1} t+O\left(t^{2}\right)$ for $t \rightarrow 0$ from (3.3.7). For this reason, we can restrict attention at this point to variations of the form

$$
\delta g(w)=\sum_{n=1}^{N} \delta g_{n} w^{n}
$$

For such $\delta g(w)$, we have

$$
\widehat{\mathcal{V}}^{-1} \delta g(w) \hat{\mathcal{V}}=\frac{\alpha_{-n}}{t^{n}}+\ldots+\frac{\alpha_{-1}}{t}+\alpha_{0}+\sum_{\nu=1}^{\infty} \alpha_{\nu} t^{\nu}
$$

by (3.3.7); the G-valued matrix functions $\alpha_{\nu}=\alpha_{\nu}(x)$ are implicitly defined through this equation. The compensating transformation $\delta h(t)$ needed to remove the pole terms is therefore 


$$
\begin{aligned}
\delta h(t) & =\frac{\alpha_{-n}}{t^{n}}+\ldots+\frac{\alpha_{-1}}{t}+\frac{1}{2}\left(\alpha_{0}+\tau\left(\alpha_{0}\right)\right)+\tau\left(\alpha_{-1}\right) t+\ldots+\tau\left(\alpha_{-n}\right) t^{n}= \\
& =\delta h_{0}+\sum_{\nu=1}^{N} \delta h_{\nu}\left(t^{\nu}+\frac{1}{t^{\nu}}\right)+\sum_{\nu=1}^{N} \delta k_{\nu}\left(t^{\nu}-\frac{1}{t^{\nu}}\right)
\end{aligned}
$$

with $\delta h_{\nu} \equiv\left(\alpha_{-\nu}+\tau\left(\alpha_{-\nu}\right)\right)$, etc.; for the definition of $\tau$, see (2.2.3). We can express the constraints $\alpha_{\nu}(x)$, or equivalently on $\delta h_{\nu}(x)$ and $\delta k_{\nu}(x)$, which follow from the definition (4.2.4), in a somewhat different form. Differentiating the left hand side of (4.2.4), we get

$$
\partial_{+}\left(\widehat{\mathcal{V}}^{-1} \delta g(w) \widehat{\mathcal{V}}\right)=\left[\widehat{\mathcal{V}}^{-1} \delta g(w) \hat{\mathcal{V}}, \widehat{\mathcal{V}}^{-1} \partial_{+} \widehat{\mathcal{V}}\right]
$$

Inserting (4.2.1) and the expansion (4.2.4) back into this expression, and comparing this with the result obtained by directly differentiating the right hand side of (4.2.4), it is straightforward to show that

$$
D_{+} \alpha_{\nu}+D_{+} \alpha_{\nu-1}+\rho^{-1} \partial_{+} \rho\left(\nu \alpha_{\nu}-(\nu-1) \alpha_{\nu-1}\right)+\left[P_{+}, \alpha_{\nu}-\alpha_{\nu-1}\right]=0
$$

where we define $\alpha_{-n-2} \equiv \alpha_{-n-1} \equiv 0$. The variation of $Q_{+}$and $P_{+}$can now be deduced from

$$
\delta\left(\widehat{\mathcal{V}}^{-1} \partial_{+} \widehat{\mathcal{V}}\right)=\partial_{+} \delta h+\left[\widehat{\mathcal{V}}^{-1} \partial_{+} \widehat{\mathcal{V}}, \delta h\right]
$$

It is essential here that the $t$-dependence of the linear system is preserved in this variation because contributions potentially violating this form of the $t$-dependence vanish by virtue of (4.2.7). Comparing the coefficients of the remaining terms in (4.2.8) and neglecting the (trivial) term with $\delta h_{0}$, one obtains

$$
\delta P_{+}=\frac{1}{2} D_{+} \delta k_{1}+\frac{1}{2} \rho^{-1} \partial_{+} \rho \delta k_{1}-\frac{1}{2}\left[P_{+}, \delta h_{1}\right]
$$

(The result for $\delta Q_{+}$is similar, but not needed here). Next, we eliminate $D_{+} \delta k_{1}$, using (4.2.7) for $\nu=2$, and repeat this procedure until all derivatives have disappeared (remember that $\delta h_{\nu}=\delta k_{\nu}=0$ for $\nu=n+1, n+2$ ). The variation of $P_{+}$is thus found to be

$$
\delta P_{+}=\rho^{-1} \partial_{+} \rho \sum_{\nu=1}^{n}(-1)^{\nu+1} \nu \delta k_{\nu}+\left[P_{+}, \sum_{\nu=1}^{n}(-1)^{\nu} \delta h_{\nu}\right]
$$

Inserting this result into the variation of (4.2.2), we see that the second term in (4.2.10) drops out, so the factor $\rho^{-1} \partial_{+} \rho$ appears on both sides and cancels out (notice that $\rho$ is not varied). Therefore,

$$
\partial_{+}\left(\lambda^{-1} \delta \lambda\right)=\sum_{\nu=1}^{n}(-1)^{\nu+1} \nu \operatorname{Tr}\left(\delta k_{\nu} P_{+}\right)
$$

In order to exhibit the right hand side as a derivative, we return to the formula (3.5.10) which, of course, remains valid for the purely bosonic theory if we put $\chi^{I}=0$. By use of the various relations given there, the relevant component of the Kac-Moody current can be written as 


$$
\widehat{\mathcal{J}}_{+}=\frac{2}{(1+t)^{2}} \frac{\partial t}{\partial w} \widehat{\mathcal{V}} P_{+} \hat{\mathcal{V}}^{-1}
$$

A short calculation now shows that the result (4.2.11) can be alternatively expressed as a contour integral in two different ways ${ }^{11}$

$$
\begin{aligned}
\partial_{+}\left(\lambda^{-1} \delta \lambda\right) & =\frac{1}{4} \oint_{t=-1} d t \frac{\partial w}{\partial t} \operatorname{Tr}\left(\hat{\mathcal{V}}^{-1} \widehat{\mathcal{J}}_{+} \hat{\mathcal{V}} \cdot \delta h(t)\right)= \\
& =-\frac{1}{2} \oint_{t=0} d t \frac{\partial w}{\partial t} \operatorname{Tr}\left(\hat{\mathcal{V}}^{-1} \hat{\mathcal{J}}_{+} \hat{\mathcal{V}} \cdot \delta h(t)\right)
\end{aligned}
$$

Therefore, integrating over the chain $\mathcal{C} \equiv \mathcal{C}_{0}+\frac{1}{2} \mathcal{C}_{-1}$, with $\mathcal{C}_{t_{0}}$ encircling the point $t_{0}$ in the complex $t$-plane once, we obtain the identity

$$
\oint_{\mathcal{c}} d t \frac{\partial w}{\partial t} \operatorname{Tr}\left(\widehat{\mathcal{J}}_{+} \cdot \delta g(w)\right)=\oint_{\mathcal{c}} d t \frac{\partial w}{\partial t} \operatorname{Tr}\left(\widehat{\mathcal{J}}_{+}\left(\delta g(w)-\hat{\mathcal{V}} \delta h(t) \hat{\mathcal{V}}^{-1}\right)\right)
$$

(for the opposite (-) chirality, one must integrate over $\mathcal{C} \equiv \mathcal{C}_{0}+\frac{1}{2} \mathcal{C}_{1}$ ). On the right hand side of (4.2.14), we can now deform the contour to a circle around $t=-1$, since the compensating transformation $\delta h(t)$ was designed precisely for the purpose of removing the pole at $t=0$ (for triangular $\widehat{\mathcal{V}}$ ). Hence,

$$
\oint_{\mathcal{C}} d t \frac{\partial w}{\partial t} \operatorname{Tr}\left(\widehat{\mathcal{J}}_{+} \cdot \delta g(w)\right)=\frac{1}{2} \oint_{t=-1} d t \frac{\partial w}{\partial t} \operatorname{Tr}\left(\widehat{\mathcal{J}}_{+}\left(\delta g(w)-\hat{\mathcal{V}} \delta h(t) \hat{\mathcal{V}}^{-1}\right)\right)
$$

Replacing the integral involving $\delta h(t)$ by $\partial_{+}\left(\lambda^{-1} \delta \lambda\right)$ according to (4.2.13) (which is the expression we set out to calculate), we arrive at

$$
\oint_{t=0} d t \frac{\partial w}{\partial t} \operatorname{Tr}\left(\widehat{\mathcal{J}}_{+} \cdot \delta g(w)\right)=-2 \partial_{+}\left(\lambda^{-1} \delta \lambda\right)
$$

In the last step, we convert the integral on the left hand side into an integral over $w$, remembering that the point $t=0$ corresponds to $w=\infty$ in the complex $w$-plane, and invoke formula (3.5.16) in the form

$$
\oint_{w=\infty} d w \operatorname{Tr}\left(\widehat{\mathcal{J}}_{+} \cdot \delta g(w)\right)=-\partial_{+}\left(\oint_{w=\infty} d w \operatorname{Tr}\left(\frac{\partial \hat{\mathcal{V}}_{\hat{\mathcal{V}}}}{\partial w} \hat{\mathcal{V}}^{-1} \cdot \delta g(w)\right)\right)
$$

In dropping the derivative operator $\partial_{+}$, we must allow for an arbitrary function depending only on $x^{-}$. This function is then fixed by repeating the above steps for $\lambda^{-1} \partial_{-} \lambda$. The final result (and the main formula of this section) is

$$
\lambda^{-1} \delta \lambda=\frac{1}{2} \oint_{w=\infty} d w \operatorname{Tr}\left(\frac{\partial \widehat{\mathcal{V}}}{\partial w} \widehat{\mathcal{V}}^{-1} \cdot \delta g(w)\right)
$$

From this formula, we can now recognize the action of the central term if we perform a second variation on $\widehat{\mathcal{V}}$ according to (4.1.7). To pick out the central term, it is sufficient to consider a $\delta g^{(2)}(w)$ of the form

11 The definition of the contour integral includes a factor $(2 \pi i)^{-1}$. 


$$
\delta g^{(2)}=\sum_{n=1}^{N} \delta g_{-n}^{(2)} w^{-n}
$$

for which, by the arguments preceding (4.2.3), the compensating $H^{\infty}$ rotation vanishes. This trick simplifies the calculation of the commutator considerably and leaves us with the result

$$
\begin{aligned}
\lambda^{-1}\left[\delta_{1}, \delta_{2}\right] \lambda & =\oint_{w=\infty} d w \operatorname{Tr}\left(\delta g^{(1)}(w) \cdot \frac{\partial}{\partial w} \delta g^{(2)}(w)\right)+ \\
& +\frac{1}{2} \oint_{w=\infty} d w \operatorname{Tr}\left(\frac{\partial \widehat{\mathcal{V}}}{\partial w} \hat{\mathcal{V}}^{-1}\left[\delta g^{(1)}(w), \delta g^{(2)}(w)\right]\right)
\end{aligned}
$$

The first contribution is the central term advertized before, while the second term is just the result expected from the Lie algebra structure of $\mathbf{G}^{\infty}$. A remarkable feature of this result, already alluded to before, is that the central term is not just a $c$-number, but a transformation operator. Already after (3.2.7), it was noted that the equations of motion admit constant rescalings of the conformal factor as an extra symmetry. The corresponding generator $\mathcal{Z}$ acts according to

$$
\lambda^{-1} \delta \lambda=\text { const }
$$

The $\mathbf{G}^{\infty}$ Lie algebra is therefore characterized by the commutation relations (with self-explanatory notation)

$$
\left[T_{m}^{a}, T_{n}^{b}\right]=f^{a b c} T_{m+n}^{c}+m \delta_{m+n, 0} \delta^{a b} \mathcal{Z}
$$

The symmetric space automorphism $\tau^{\infty}$ can be generalized to the centrally extended algebra [10]. Using (3.3.10) it is easy to check that (4.2.22) is invariant under

$$
\tau^{\infty}\left(T_{m}^{a}\right)=\tau\left(T_{-m}^{a}\right), \tau^{\infty}(\mathcal{Z})=-\mathcal{Z}
$$

This means that, in contrast to $\mathbf{G}^{\infty}$, the $\tau^{\infty}$ invariant subalgebra $\mathbf{H}^{\infty}$ does not possess a central extension.

The appearance of the operator $\mathcal{Z}$ in the Kac Moody algebra (4.2.20) suggests that the conformal factor $\lambda$ should be included into an enlarged version of the " $\infty$-bein" $\widehat{\mathcal{V}}$. In accordance with this idea, it was proposed in [10] to consider pairs $(\widehat{\mathcal{V}}, \lambda)$ with the multiplication rule

$$
\left(\widehat{\mathcal{V}}_{1}, \lambda_{1}\right) \circ\left(\widehat{\mathcal{V}}_{2}, \lambda_{2}\right)=\left(\widehat{\mathcal{V}}_{1} \widehat{\mathcal{V}}_{2}, \lambda_{1} \lambda_{2} \exp \Omega\left(\widehat{\mathcal{V}}_{1}, \widehat{\mathcal{V}}_{2}\right)\right)
$$

where $\Omega\left(\widehat{\mathcal{V}}_{1}, \widehat{\mathcal{V}}_{2}\right)$ is a group two-cocycle (an introduction for physicists is given in [52]). This cocycle is only determined up to a coboundary, which can be absorbed into a multiplicative redefinition of $\lambda$. The above multiplication rule correctly implements the central extension of the Kac Moody algebra $\mathbf{G}^{\infty}$ at the level of the Kac Moody group $G^{\infty}$. Denoting the centrally extended version of $G^{\infty}$ by $G_{c e}^{\infty}$, it is clear that the coset space $G_{c e}^{\infty} / H^{\infty}$ is related to the space of solutions of (3.2.5) and (4.2.2) in the same way as $G^{\infty} / H^{\infty}$ was related to the solutions of (3.2.5) alone. The Geroch group is now properly identified as $G_{c e}^{\infty}$. 
It is perhaps worth emphasizing that through the enlargement of the original " $\infty$-bein" to a new entity also containing the conformal factor, the unification of symmetries, which is observed in the reduction from higher dimensions, for the first time touches upon the unphysical gravitational degrees of freedom (while, incidentally, the last remnant of local Lorentz invariance on the zweibein has disappeared). This indicates that any further unification of symmetries beyond $G^{\infty}$ will involve the boost generators.

As pointed out in [10], the automorphism $\tau^{\infty}$ can generalized to the centrally extended Kac Moody group. The analog of (3.3.10) is

$$
\tau^{\infty}(\widehat{\mathcal{V}}, \lambda)=\left(\tau^{\infty} \hat{\mathcal{V}}, \lambda^{-1}\right)
$$

We can now repeat the argument which was used in (3.4.2) to prove the $x$ independence of the monodromy matrix and show that

$$
\widetilde{\mathcal{M}} \equiv(\widehat{\mathcal{V}}, \lambda) \circ \tau^{\infty}(\widehat{\mathcal{V}}, \lambda)^{-1}=\left(\widehat{\mathcal{V}}^{\infty} \hat{\mathcal{V}}^{-1}, \lambda^{2} \exp \Omega\left(\widehat{\mathcal{V}}, \tau^{\infty} \widehat{\mathcal{V}}^{-1}\right)\right)
$$

is likewise $x$-independent. Thus, the second entry on the right hand side must be constant, which immediately leads to a beautiful formula for the conformal factor in terms of $\hat{\mathcal{V}}[10]$, viz.

$$
\ln \lambda=-\frac{1}{2} \Omega\left(\widehat{\mathcal{V}}, \tau^{\infty} \hat{\mathcal{V}}^{-1}\right)+\text { const }
$$

Unfortunately, the practical usefulness of this result is limited by the scarcity of explicit formulas for group cocycles [52]. Nonetheless, there are some special cases where an explicit computation is possible and (4.2.27) can be "tested". One is the colliding plane wave solution reconstructed in section 3.4 , for which the matrix $\widehat{\mathcal{V}}$ is diagonal due to the collinear polarization of the waves. By use of the formulas given in appendix B of [10], (4.2.27) reduces to

$$
\begin{aligned}
\ln \lambda & =-\frac{1}{2} \oint d t \frac{\partial}{\partial t} \ln \left(\sqrt{-\frac{t_{1}}{t_{2}}} \frac{t-t_{1}}{t-t_{2}}\right) \ln \left(\sqrt{-\frac{t_{1}}{t_{2}}} \frac{1-t t_{1}}{1-t t_{2}}\right)= \\
& =+\frac{1}{2} \ln \frac{\left(1-t_{1} t_{2}\right)^{2}}{\left(1-t_{1}^{2}\right)\left(1-t_{2}^{2}\right)}
\end{aligned}
$$

This agrees with (3.4.13) after re-insertion of the factor $\left(\partial_{+} \rho\right)^{-\frac{1}{2}}\left(\partial_{-} \rho\right)^{-\frac{1}{2}}$ and adjustment of the integration constant.

I conclude this section with some comments on the supersymmetric theory. As already mentioned, the above derivation of the central term is more general than previous ones in that it also works for supergravity (the method of commuting an Ehlers with a Matzner-Misner transformation and evaluating its effect on the conformal factor $[17,10]$ does not obviously generalize beyond $S L(2, \mathbb{R})$, and is certainly insufficient for supergravity). Remarkably, formula (4.2.18) for the variation of the conformal factor remains valid as it stands, although the intermediate steps are different and more cumbersome due to the extra fermionic terms in (4.2.2) and the appearance of higher order poles in the linear system. Amongst other things, one must work out the action 
of the compensating duality rotation on the fermions. In a chiral basis (with $\chi_{+} \equiv \frac{1}{2} \gamma_{-} \gamma_{+} \chi$ and $\chi_{-} \equiv \frac{1}{2} \gamma_{+} \gamma_{-} \chi$, etc.), one finds that the chiral components of $\chi$ transform as eigenstates under the action of $\delta h(x ; t)$ at $t= \pm 1$, viz.

$$
\delta \chi_{+}=\delta h(x ;-1) \chi_{+}, \delta \chi_{-}=\delta h(x ;+1) \chi_{-}
$$

This is, of course, not an unexpected result in view of the fact that the fermionic analogs of the "dual potentials" in (3.3.7) are still missing.

\subsection{Hints of More Symmetries}

The foregoing analysis has revealed an impressive array of "hidden symmetries" in Einstein's theory and its various generalizations. Nevertheless, there are still more symmetries even in the purely bosonic theory, and it seems like we have only had a glimpse of the full structure so far! As discovered only very recently, one can realize a Virasoro algebra on the space bosonic solutions $[49,18]$. It is most simply defined through its action on $\widehat{\mathcal{V}}$

$$
\widehat{\mathcal{V}} \rightarrow \delta w \frac{\partial \hat{\mathcal{V}}}{\partial w}
$$

where $\delta w=f(w)$ with an arbitrary meromorphic function $f(w)$. Expanding $f(w)$ in powers of $w$, we have the following correspondence with the well-known Virasoro generators

$$
L_{m}: \widehat{\mathcal{V}} \rightarrow-w^{m+1} \frac{\partial \widehat{\mathcal{V}}}{\partial w}
$$

To keep $\widehat{\mathcal{V}}$ in the triangular gauge, a compensating $H^{\infty}$ rotations is again necessary. However, there is a further subtlety now. To maintain the pole structure of the linear system, a compensating conformal coordinate transformation of the type (3.2.4) is also needed. More precisely, one demands that

$$
\delta t=\delta w \frac{\partial t}{\partial w}+\delta x^{+} \partial_{+} t+\delta x^{-} \partial_{-} t
$$

should be regular as $t \rightarrow \pm 1$, so no higher order poles are introduced through the transformation (4.3.1) into the linear system (this compensating transformation is also necessary to take care of the extra contribution $\delta\left(\rho^{-1} \partial_{+} \rho\right) \neq 0$ in the variation of (4.2.2)). It is not difficult to prove that the generators $L_{m}$ indeed satisfy a Virasoro algebra, and that the commutator of a Virasoro and a $\mathbf{G}^{\infty}$ transformation gives expected result

$$
\left[L_{m}, T_{n}^{a}\right]=-n T_{m+n}^{a}
$$

Finally, the conformal factor also transforms under the Virasoro algebra, and its variation can be determined from (4.2.2) as before.

The presence of the extra symmetry (4.3.1) suggests that the coset space $G_{c e}^{\infty} / H^{\infty}$ may not be enough to properly characterize the space of solutions, since it does not take the extra symmetry (4.3.1) into account. Likewise, the 
subgroup $H^{\infty}$ should be extended so as to accomodate the conformal coordinate transformations (3.2.4). This, too, may not be enough for the supersymmetric theory because it was shown in [20] that after re-introducing the gravitino components into the linear system (3.5.11), the local superconformal invariance of the theory could be expressed as a Grassmann valued $H^{\infty}$ gauge transformation (plus a compensating conformal coordinate transformation, see the appendix). All of this hints at the existence of an even larger coset space in terms of an as yet unknown quotient $G^{\infty \infty} / H^{\infty \infty}$. In view of our ignorance about $G^{\infty \infty}$ and $H^{\infty \infty}$, it is advisable to collect the available information. Designating the associated Lie algebras by $\mathbf{G}^{\infty \infty}$ and $\mathbf{H}^{\infty}$, respectively, we know that the set of rigid symmetry generators must contain $\mathbf{G}^{\infty}, \mathcal{Z}$ and the Virasoro algebra $\operatorname{Vir}(w)$. Thus

$$
\mathbf{G}^{\infty \infty} \supset \mathbf{G}^{\infty} \oplus \mathcal{Z} \oplus \operatorname{Vir}(w)
$$

For the gauge symmetries, we have in addition to $\mathbf{H}^{\infty}$ the generators of the conformal coordinate transformations (but no central term!), or

$$
\mathbf{H}^{\infty \infty} \supset \mathbf{H}^{\infty} \oplus \operatorname{Vir}\left(x^{+}\right) \oplus \operatorname{Vir}\left(x^{-}\right)
$$

where $\operatorname{Vir}\left(x^{ \pm}\right)$stands for the algebra of conformal coordinate transformations (3.2.4) depending on $x^{+}$or $x^{-}$. What could the algebra $\mathbf{G}^{\infty}$ be? A glance at the table of Cartan Kac Moody Lie algebras shows that, beyond the known affine Kac Moody algebras, there remain only the so-called hyperbolic algebras $[23,53]$. It was in fact this observation which led to the conjecture that such hyperbolic algebras should emerge in the reduction to one dimension [24]. In the appendix, I will argue that this last step should involve a chiral reduction, so one of the summands $\operatorname{Vir}\left(x^{ \pm}\right)$should be omitted from (4.3.6). Nonetheless, even without actually considering this reduction in detail, we can adduce some evidence for the correctness of the conjecture by taking a closer look at (4.3.5). The point here is that the extra generators $\mathcal{Z}$ and $L_{0} \in \operatorname{Vir}(w)$ identified in (4.3.5) are precisely the generators needed to enlarge the Cartan subalgebra of $\mathbf{G}$ to that of its hyperbolic extension! This is most easily understood in the language of string vertex operators. In the vertex operator construction of Kac Moody algebras [51,54], the Cartan subalgebra is always spanned by the zero mode string momentum operators $\oint \varepsilon_{\mu} \partial X^{\mu}$. Choosing the polarization vector $\varepsilon_{\mu}$ space-like, one gets the Cartan subalgebra of the finite dimensional Lie algebra $\mathbf{G}$. Taking $\varepsilon_{\mu}$ to be one of the remaining two light-like vectors, on the other hand, one obtains just the two operators $\mathcal{Z}$ and $L_{0}$ above (these operators are called $k$ and $d$ in [51]). Observe that the metric in root space has the required signature $(-+\ldots+)$. In fact, one can embed the whole Virasoro algebra $\operatorname{Vir}(w)$ into the hyperbolic extension by considering vertex operators of the form $\oint: \bar{\varepsilon} \partial X \exp (i m \varepsilon X)$;, where $\varepsilon_{\mu}$ and $\bar{\varepsilon}_{\mu}$ are the two light-like vectors obeying $\varepsilon \cdot \bar{\varepsilon}=1$, and $m \in \mathbb{Z}$.

Obviously, these considerations do not suffice to really prove that $\mathbf{G}^{\infty}$ is the hyperbolic extension of $\mathbf{G}$, even after reduction to one dimension. Moreover, it appears that the inclusion of fermions is absolutely indispensable if one is to get the full hyperbolic algebra. The reason for this expectation is related to 
the following puzzle, which has been carefully kept under the rug so far. Up to this point, I have pretended that the addition of fermions is hardly more than a technicality, resulting in higher order poles in the linear system and the like, but not fundamentally altering the structure of the theory. For this reason, we have so far mainly dealt with the bosonic theory, identifying the coset space $G_{c e}^{\infty} / H^{\infty}$ with the bosonic solutions. Arguments were given at the end of section 3.4, that these solutions indeed cover the whole coset space. What happens when we switch on the fermionic degrees of freedom? Surely, the space of solutions must be enlarged in one way or another, if the bosonic solutions fill out the coset space already by themselves, leaving "no more room" for the fermions ${ }^{12}$. The folklore line of reasoning might now entice one into believing that cosets should simply be replaced by super-cosets, but here we encounter trouble: once the local supersymmetry has been eliminated by fixing a gauge, no rigid supersymmetry remains that could act on a super coset space. Moreover, in many cases of interest (e.g. $\mathbf{G}=E_{8}$ and $\mathbf{G}^{\infty}=E_{9}$ ), a super-extension of the algebra $\mathbf{G}^{\infty}$ does not exist [22]! There seems to be only one way out of this impasse: the space of combined bosonic and fermionic solutions must be related to the hyperbolic extension of the affine Kac-Moody algebra $\mathbf{G}^{\infty}$. The missing generators on the right hand side of (4.3.5) would then correspond to transformations mixing bosonic and fermionic solutions. These conclusions seem to concur with the analysis of [55], where the the hyperbolic algebra extending $A_{1}^{(1)}$ was shown to exhibit a graded structure reminiscent of a fermionic Fock space. Could it be that the new "supersymmetry transformations", which have apparently no analog in higher dimensions, are related to the hyperbolic generators associated with negative norm roots, for which no manageable representation has been found so far?

Acknowledgements: I would like to thank the organizers for inviting me to this very pleasant meeting and $\mathrm{P}$. Aichelburg and $\mathrm{C}$. Isham for some interesting discussions related to this work. I am especially grateful to P. Breitenlohner and D. Maison for having contributed to these lectures through many stimulating (and occasionally frustrating) discussions.

\section{Appendix: Maximally Extended Supergravity in One Dimension}

This appendix deals with maximally extended $(N=16)$ supergravity in one dimension. The notation and conventions are taken from [19-21], which readers are advised to consult for further explanations and details. In addition to the gauge conditions (3.2.2) and (3.5.1), I will employ the light-cone conventions already used in the main body of this article, in particular $\chi_{+} \equiv \frac{1}{2} \gamma_{-} \gamma_{+} \chi$ and $\chi_{-} \equiv \frac{1}{2} \gamma_{+} \gamma_{-} \chi$ for the fermionic fields (in fact, we could introduce "onecomponent spinors" at this point, but I will not do so). Although the results below are deficient in that they only represent the very first steps in the search

12 Nor does the coset space of the bosonic theory become larger after the reduction to one dimension, which again indicates that the reduction of the bosonic theory without fermions will not lead to the desired enhancement of symmetry. 
for more symmetries, they might nonetheless be useful at some later stage, and this is my reason for including them in these lectures. Contrary to the prescription followed in chapters 2 and 3 , it is proposed here to perform the dimensional reduction by dropping the dependence on one of the light-cone coordinates $x^{ \pm}$ rather than $x^{0}$ or $x^{1}$. In other words, the last step in the dimensional reduction should involve a chiral truncation, so instead of (2.1.1)

$$
\phi\left(x^{0}, x^{1}\right) \longrightarrow \phi\left(x^{+}\right) \text {or } \phi\left(x^{-}\right)
$$

for all fields. One advantage of this procedure is that, unlike in the ordinary dimensional reduction, the equations of motion are enforced by this truncation. As a consequence, the algebra of local supersymmetry transformations closes without further ado (i.e. off-shell) on all fields with the exception of $\psi_{2+}^{I}$, where a term proportional to the supersymmetry current $J_{+}^{I}$ remains in the commutator.

Imposing (A.1) in the two-dimensional equations of motion immediately implies that certain chiral components vanish. More explicitly, using the notation of $[19,20]$, one has

$$
\chi_{-}^{\dot{A}}=\psi_{-}^{I}=\psi_{2-}^{I}=Q_{-}^{I J}=P_{-}^{A}=0
$$

As already mentioned, the remaining $(+)$ components of the "matter fields" then satisfy their respective equations of motion automatically. Observe that through (A.2) half of the physical degrees of freedom are eliminated. This would not have been the case for an ordinary reduction, where we would have ended up with $N=32$ locally supersymmetric quantum mechanics instead. The only equations of motion that must still be imposed are those obtained by varying the traceless components of the two-metric and the gravitino, which were eliminated through the gauge conditions (3.2.2) and (3.5.1), respectively. These are just the generators of the remaining local invariances, namely the supersymmetry current $J_{+}^{I}$ and the energy momentum tensor $T_{++}$.

The full supersymmetry variations of chiral $N=16$ supergravity can be derived by truncation of the corresponding transformations in [21] and are characterized by the chiral transformation parameter

$$
\epsilon^{I}=\epsilon_{-}^{I}\left(x^{+}\right) \text {i.e. } \epsilon_{+}^{I}=0
$$

In addition to the terms obtained directly from [21], a compensating $S O(1,1)$ rotation necessary to preserve the diagonality of the zweibein must be taken into account. Furthermore, it should be remembered that the gravitino components below are defined from $\psi_{a}^{I}=e_{a}^{m} \psi_{m}^{I}$ (flat indices), and that the second order spin connections in the gravitino variations have been written out explicitly. Keeping track of all these contributions, one arrives at the the supersymmetry variations

$$
\begin{gathered}
\rho^{-1} \delta \rho=-\bar{\epsilon}_{-}^{I} \psi_{2+}^{I} \quad, \quad \lambda^{-1} \delta \lambda=i \bar{\epsilon}_{-}^{I} \psi_{+}^{I} \\
\mathcal{V}^{-1} \delta \mathcal{V}=\bar{\epsilon}_{-}^{I} \chi_{+}^{\dot{A}} \Gamma_{A \dot{A}}^{I} Y^{A}
\end{gathered}
$$

for the bosons, and 


$$
\begin{gathered}
\delta \chi_{+}^{\dot{A}}=\frac{i}{2} \lambda^{-1} \gamma_{-} \epsilon_{-}^{I} \Gamma_{A \dot{A}}^{I} \widehat{P}_{+}^{A}+\frac{i}{2} \chi_{+}^{\dot{A}} \cdot \bar{\epsilon}_{-}^{J} \psi_{+}^{J} \\
\delta \psi_{+}^{I}=\frac{1}{2} \lambda^{-1} \gamma_{-}\left(D_{+}+\frac{1}{2} \lambda^{-1} \partial_{+} \lambda\right) \epsilon_{-}^{I}-\frac{3 i}{2} \psi_{+}^{I} \cdot \bar{\epsilon}_{-}^{J} \psi_{+}^{J}+\frac{i}{8} \gamma_{-} \epsilon_{-}^{J} \cdot \bar{\chi}_{+} \gamma_{+} \Gamma^{I J} \chi_{+} \\
\delta \psi_{2+}^{I}=-\frac{i}{2} \lambda^{-1} \rho^{-1} \partial_{+} \rho \gamma_{-} \epsilon_{-}^{I}+\frac{i}{2} \psi_{2+}^{I} \cdot \bar{\epsilon}_{-}^{J} \psi_{+}^{J}+\frac{1}{2} \gamma_{-} \epsilon_{-}^{J} \cdot \bar{\psi}_{2+}^{I} \gamma_{+} \psi_{2+}^{J}+ \\
+\frac{i}{2} \gamma_{-} \epsilon_{-}^{I} \cdot \bar{\psi}_{+}^{J} \gamma_{+} \psi_{2+}^{J}+\frac{1}{4} \gamma_{-} \epsilon_{-}^{J} \cdot \bar{\chi}_{+} \gamma_{-} \Gamma^{I J} \chi_{+}
\end{gathered}
$$

for the fermions with

$$
\widehat{P}_{+}^{A} \equiv P_{+}^{A}-\lambda \Gamma_{A \dot{A}}^{I} \bar{\chi}_{+}^{\dot{A}} \gamma_{+} \psi_{+}^{J}
$$

On all fields but $\psi_{2+}^{I}$, this algebra closes "off shell". With some heavy Fierzing (see the appendix of [21] for the necessary technology), one finds

$$
\left[\delta\left(\epsilon_{1}\right), \delta\left(\epsilon_{2}\right]=\delta_{\text {conf }}\left(\xi^{\prime}\right)+\delta_{S U S Y}\left(\epsilon^{\prime}\right)+\delta_{S O(16)}\left(\omega^{\prime}\right)\right.
$$

with the parameters

$$
\begin{gathered}
\xi_{-}^{\prime}=i \lambda^{-1} \bar{\epsilon}_{2-}^{I} \gamma_{-} \epsilon_{1-}^{I} \\
\epsilon_{-}^{\prime I}=-i \gamma_{+} \psi_{+}^{I} \cdot \bar{\epsilon}_{2-}^{K} \gamma_{-} \epsilon_{1-}^{K}+\frac{2}{2} \gamma_{+} \psi_{+}^{K} \cdot \epsilon_{2-}^{(K} \gamma_{-} \epsilon_{1-}^{I)} \\
{\omega^{\prime}}^{I J}=\xi_{-}^{\prime} Q_{+}^{I J}+\frac{1}{4} \bar{\epsilon}_{2-}^{K} \gamma_{-} \epsilon_{1-}^{K} \cdot \bar{\chi}_{+} \gamma_{+} \Gamma^{I J} \chi_{+}+\bar{\epsilon}_{2-}^{K} \gamma_{-} \epsilon_{1-}^{I I} \cdot \bar{\chi}_{+} \gamma_{+} \Gamma^{\eta K} \chi_{+}
\end{gathered}
$$

On the gravitino component $\psi_{2+}^{I}$, one gets an extra contribution in the commutator

$$
\left[\delta\left(\epsilon_{1}\right), \delta\left(\epsilon_{2}\right)\right] \psi_{2+}^{I}=\ldots-i \bar{\epsilon}_{2-}^{J} \gamma_{-} \epsilon_{1-}^{J} \cdot J_{+}^{I}+i \bar{\epsilon}_{2-}^{(I} \gamma_{-} \epsilon_{1-}^{J)} \cdot J_{+}^{J}
$$

where the supercurrent $J_{+}^{I}$ (the gravitino equation of motion) is given by

$$
\begin{aligned}
J_{+}^{I}= & \lambda^{-1} \rho^{-1} D_{+}\left(\rho \psi_{2+}^{I}\right)-\frac{1}{2} \lambda^{-2} \partial_{+} \lambda \psi_{2+}^{I}+i \lambda^{-1} \rho^{-1} \partial_{+} \rho \psi_{+}^{I}-\lambda^{-1} \Gamma_{A \dot{A}}^{I} \chi_{+}^{\dot{A}} \widehat{P}_{+}^{A}- \\
& -i \bar{\psi}_{+}^{K} \gamma_{+} \psi_{2+}^{K} \cdot \psi_{+}^{I}-\frac{1}{2} \bar{\chi} \gamma_{+} \gamma_{+} \Gamma^{I J} \chi_{+} \cdot \psi_{+}^{J}-\frac{i}{4} \bar{\chi}_{+} \gamma_{+} \Gamma^{I J} \chi_{+} \cdot \psi_{2+}^{J}
\end{aligned}
$$

Thus, on $\psi_{2+}^{I}$, the algebra closes only if $J_{+}^{I}=0$. As explained in section 2.3 , this is just the condition defining the physical sector in phase space. The above results can be streamlined somewhat by rescaling the fermion fields according to

$$
\chi \rightarrow \lambda^{\frac{1}{2}} \chi \quad, \quad \psi_{+}^{I} \rightarrow \lambda^{\frac{1}{2}} \psi_{+}^{I} \quad, \quad \psi_{2+}^{I} \rightarrow \lambda^{\frac{1}{2}} \psi_{2+}^{I}
$$

and the supersymmetry transformation parameter according to

$$
\epsilon_{-}^{I} \rightarrow \lambda^{-\frac{1}{2}} \epsilon_{-}^{I}
$$

(The supersymmetry current is rescaled by a factor $\lambda^{\frac{3}{2}}$.) In the remainder of this appendix I will use rescaled fields.

The $(+)$ component of the linear system for $N=16$ supergravity takes the form

$$
\widehat{\mathcal{V}}^{-1} \partial_{+} \widehat{\mathcal{V}}=\frac{1}{2} \mathcal{Q}_{+}^{I J}(t) X^{I J}+\mathcal{P}_{+}^{A}(t) Y^{A}
$$

with 


$$
\begin{aligned}
\mathcal{Q}_{+}^{I J}(t)=Q_{+}^{I J}+ & \frac{t}{(1+t)^{2}}\left(-i \bar{\chi}_{+} \gamma_{+} \Gamma^{I J} \chi_{+}+8 \bar{\psi}_{2+}^{[I} \gamma_{+} \psi_{+}^{J}\right) \\
& -16 \frac{t^{2}}{(1+t)^{4}} i \bar{\psi}_{2+}^{I} \gamma_{+} \psi_{2+}^{J}
\end{aligned}
$$

and

$$
\mathcal{P}_{+}^{A}(t)=P_{+}^{A}+2 \frac{t(1-t)}{(1+t)^{3}} i \Gamma_{A \dot{A}}^{I} \bar{\psi}_{2+}^{I} \gamma_{+} \chi_{+}^{\dot{A}}
$$

Of course, (A.13) loses some of its lustre in one dimension since the compatibility condition (3.3.3) is trivially satisfied for $x^{-}$-independent $\hat{\mathcal{V}}$. Nevertheless, the matrix $\widehat{\mathcal{V}}$ remains useful as it describes all the degrees of freedom in a unified manner. In [20], it was pointed pointed out that the local supersymmetry transformations can be re-expressed as a Grassmann-valued $H^{\infty}$ gauge transformation

$$
\widehat{\mathcal{V}}^{-1} \delta \widehat{\mathcal{V}}=\frac{1}{2} S^{I J}(t) X^{I J}+S^{A}(t) Y^{A}
$$

with

$$
S^{I J}(t)=-\frac{8 t}{(1+t)^{2}} \bar{\epsilon}_{-}^{[I} \psi_{2+}^{J]} \quad, \quad S^{A}(t)=\frac{1-t}{1+t} \Gamma_{A \dot{A}}^{I} \bar{\epsilon}_{-}^{I} \chi_{+}^{\dot{A}}
$$

provided the spectral parameter is also varied according to

$$
t^{-1} \delta t=-\frac{1-t}{1+t} \bar{\epsilon}_{-}^{I} \psi_{2+}^{I}
$$

One finds

$$
\begin{aligned}
\frac{1}{2} \delta \mathcal{Q}_{+}^{I J}(t) X^{I J}+\delta \mathcal{P}_{+}^{A}(t) Y^{A}= & \partial_{+}\left(\hat{\mathcal{V}}^{-1} \delta \widehat{\mathcal{V}}\right)+\left[\hat{\mathcal{V}}^{-1} \partial_{+} \widehat{\mathcal{V}}, \hat{\mathcal{V}}^{-1} \delta \hat{\mathcal{V}}\right]- \\
& -\frac{8 t}{(1+t)^{2}} \bar{\epsilon}_{-}^{I} J_{+}^{J} \cdot \frac{1}{2} X^{I J}
\end{aligned}
$$

It should come as no surprise that the results obtained by varying the right hand side of (A.13) by use of (A.4) and (A.5), and the one obtained by substituting (A.16) into (A.19) again only agree on the physical subspace $J_{+}^{I}=0$. Agreement is likewise obtained for the cubic fermionic terms in the supersymmetry variations (A.4) and (A.5), as I have explicitly verified (this consistency check was not performed in [20]). As already remarked in [20], the result (A.18) is suggestive of some hierarchy of $H^{\infty}$ (or $H^{\infty}$ ?) gauge transformations, of which local supersymmetry would only be a tiny part. Finally, the supersymmetry variation of the spectral parameter (A.18) can be replaced by a conformal transformation with parameter $\xi_{\text {- defined through }}$

$$
\partial_{+} \rho \xi_{-}=-\rho \bar{\epsilon}_{-}^{I} \psi_{2+}^{I}
$$

Let me end this appendix (and the lectures) on a speculative note. The potentially most important reason for advocating a chiral reduction is that the $N=16$ theory described here may give rise to a new dual model which would be related to $D=11$ supergravity [56] in the same way as the ordinary 
closed superstring [39] is related to $N=2$ supergravity in ten dimensions ${ }^{13}$. Of course, it is well known that the conventional superconformal theories defining the various superstrings stop at $N=4$ [57]. On the other hand, $N=16$ supergravity is not a conventional superconformal theory. Among other things, this is evident from the fact that the algebra (A.7) is "soft" (i.e. the structure constants are field dependent). Despite these differences, the similarities with string theory are intriguing. The usual (super-)Virasoro generators are now replaced by the requirement that (A.10) and the energy momentum tensor (alias the Wheeler-DeWitt operator)

$$
T_{++}=\lambda^{-1} \partial_{+} \lambda \partial_{+} \rho-\frac{1}{2} \partial_{+}^{2} \rho-\frac{1}{2} \rho P_{+}^{A} P_{+}^{A}+\frac{2}{2} \rho \bar{\chi}_{+}^{\dot{A}} \gamma_{+} D_{+} \chi_{+}^{\dot{A}}+\ldots
$$

annihilate the physical states. As one can see, the role of the unphysical target space coordinates $X^{ \pm}$and their fermionic partners is now played by the fields $\rho, \lambda, \psi_{+}^{I}$ and $\psi_{2+}^{I}$, which originate from the higher-dimensional vielbein and gravitino. It is encouraging that, with the assumption that the bosonic and fermionic creation operators are related to the Fourier modes of $P_{+}^{A}\left(x^{+}\right)$and $\chi_{+}^{A}\left(x^{+}\right)$and that the groundstate of this "superstring" is just the massless multiplet of $D=11$ supergravity (consisting of the 44 and 84 representations of $S O(9)$ in the bosonic sector, and the 128 representation in the fermionic sector), we can adapt the arguments given in [58] to show that the states of the first excited level can be assembled into multiplets of $S O(10)$ ! The full $S O(1,10)$ Lorentz algebra would then have to be contained in the algebra $\mathbf{H}^{\infty}$ in the same way as its transverse $S O(9)$ subalgebra is contained in $\mathbf{H}^{\infty}$.

\section{References}

Solutions of Einstern's Equations: Techniques and Results, ed. by C. Hoenselaers and W. Dietz (Springer, Berlin 1984)

2 T Kaluza: Sitzungsberichte der preussischen Akademie der Wissenschaften 1921, 966 , O. Klein. Z Phys. 37, 895 (1926)

3 J Ehlers. Dissertation, Hamburg University (1957),

G. Neugebauer and D Kramer: Annalen der Physik 24, 62 (1969),

R. Geroch: J. Math. Phys. 12, 918 (1971),

D Maison. Gen Rel Grav 10,717 (1979)

4 R Geroch J.Math Phys 13, 394 (1972)

$5 \quad$ W Kinnersley and D Chitre J Math Phys. 18,1538 (1977), 19, 1926 and 2037 (1978)

13 A very similar proposal was already made in [19], where it was suggested to interpret the $d=2, N=16$ theory as a superstring. Apart from the technical difficulties of realizing this idea in practice, there is a problem with the number of physical degrees of freedom The $d=2$ theory still has both the left and right moving degrees of freedom (corresponding to pure supergravitational radiation as opposed to the solitonic excitations of the type encountered in section 3.4), and would therefore give rise to a kind of closed superstring. This would imply the factornzability of the states into products of right and left moving states. However, the massless multiplet of $D=11$ supergravity does not admit such a factorization unlike the corresponding nultiplet of $D=10, N=2$ supergravity. Clearly, this problem does not arise for the undexterous reduction discussed here. 
B.K.Harrison: Phys.Rev.Lett. 41, 1197 (1978);

G. Neugebauer: J.Phys. A12, L67 (1979)

V.A. Belinskii and V.E. Sakharov: Zh.Eksp.Teor.Fiz. 75,1955 (1978); 77, 3 (1979)

D Maison· Phys.Rev Lett. 41, 521 (1978)

F. Ernst and I. Hauser: Phys.Rev. D20, 362 (1979)

K Ueno and Y. Nakamura: Phys. Lett. 117B, 208 (1982)

P. Brettenlohner and D. Maison in [1] and Ann Inst. Poincare 46, 215 (1987)

K Khan and R. Penrose: Nature 229, 286 (1970);

P. Szekeres' J.Math.Phys 13, 286 (1972);

$M$ Nutku and $M$. Halil Phys.Rev.Lett. 39,1379 (1977);

V. Ferrari: in General Relativity and Gravitation 1989, ed. by N. Ashby, D.F.Bartlett and W. Wyss (Cambridge University Press 1990)

V. Ferrari and J. Ibanez: Gen Rel.Grav. 19, 405 (1987)

F. Ernst and I Hauser: J.Math Phys. 28, 2155 (1987); 28, 2951 (1987); 29, 581 (1988)

Unification of The Fundamental Particle Interactions, ed. by S. Ferrara, J. Ellis and P. van Nieuwenhuizen (Plenum Press, New York, 1980);

Unification of The Fundamental Particle Interactions II, ed. by S. Ferrara and J. Ellis (Plenum Press, New York, 1983),

Supersymmetry and Supergravity '82, ed. by S. Ferrara, J G. Taylor and P. van Nreuwenhuizen (World Scientıfic, Singapore, 1983)

E Cremmer and B Julia. Nucl.Phys 159B,141 (1979)

B. Julia in Superspace and Supergravily, ed by S W Hawkıng and M Rocek (Cambridge University Press, 1980)

B Julia in Johns Hopkzns Workshop on Current Problems in Particla Physzcs: Unzfied Freld Theorzes and Beyond, Johns Hopkins University, Baltimore (1981)

D. Maison. preprint MPI-PAE/PTh 80/88 (1988)

H. Nicolai: Phys. Lett. 194, 402 (1987)

H Nicolai and N.P. Warner: Comm.Math.Phys. 125, 384 (1989)

$\mathrm{N}$ Marcus and J H Schwarz. Nucl Phys B228, 145 (1983)

V.G Kac: Adv. in Math. 26, 8 (1977)

V G. Kac Infinile Dimensional Lie Algebras, (Birkhauser, Boston, 1983)

B Julia. in Vertex Operators in Mathematics and Physics, ed. by J Lepowsky, S Mandelstam and I. Singer (Springer Verlag, Heidelberg, 1984)

E. Del Giudice, P DıVecchia and S Fubini Amu Phys 70, 378 (1972);

J Scherk Rev Mod Phys. 47,123 (1975)

P. Breitenlohner, D Maison and G W Gibbons Comm Math Phys. 120, 295 (1988)

D Z Freedman, S Ferrara and P. van Nieuwenhuizen Phys.Rev. B13, 3214 (1976);

S. Deser and B Zumino: Phys Lett. 62B, 335 (1976)

P. van Nieuwenhuizen. Phys. Rep 68,189 (1981),

D.Z Freedman and B. de Wit. in Supersymmetry, ed. by K. Dietz, R. Flume, G. v. Gehlen and V Rittenberg (Plenum Press, New York, 1985)

B. de Wit and H, Nicolai. Nucl Phys 274, 363 (1986)

S Coleman, J Wess and B Zummo. Phys Rev 177, 2239 (1969)

$H$ Eichenherr and $M$ Forger: Nucl Phys B155, 381 (1979)

$\mathrm{C}$ Misner, $\mathrm{K}$ Thorne and J A Wheeler Gravitation (Freeman, San Francisco 1973);

C. Isham in General Relativnly and Grantalion, Cambridge University Press' (1987)

H Nicolas. Nucl Phys. B353, 493 (1991)

P A M Dirac. Lectures on Quantum Mechanıcs, Academı Press, London (1967)

J.A Wheeler: in Relativity, Groups and Topology, ed, by C. DeWitt and B. DeWitt (Gordon and Breach, 1964);

B. DeWitt. Phys. Rev. 160,113 (1967); 162,1195 (1967)

V. Husain and L. Smolin. Nucl.Phys. B327, 205 (1989)

A Ashtekar. New Perspectıves in Canonıcal Gravity (Bibliopolis, Naples, 1988)

H. Nicolal Phys.Lett 235B,195 (1990)

J.H Schwarz: Phys Rep. 89, 223 (1982)

M. Luscher and K Pohlmeyer: Nucl Phys. B137,46 (1978)

M.C Davies, PJ. Houston, J M Lemaas and A J Macfarlane: Phys.Lett 119B,187 (1982),

H.J de Vega, H Eichenherr and J M Maillet Comm.Math.Phys 92, 507 (1984)

F. Ernst: Phys Rev. 167, 1175 (1968) 
43 F. Ernst and I. Hauser: J Math Phys. 30, 872 (1989)

44 K. Pohlmeyer: Comm Math.Phys. 46, 207 (1976)

45 W. Zakrzewski. Durham preprint DTP-87/3 (1987)

46 D. Maison and P. Breitenlohner: MPI report, unpublished

47 H Eichenherr and M Forger: Comm. Math Phys. 82, 227 (1981)

48 P.C. Aichelburg and R Güven Phys Rev.Lett 51, 1613 (1983)

49 Bo-Yu Hou and Wel Li Lett.Math.Phys 13, 1 (1987)

50 F. Ernst and I Hauser J Math Phys 22,1 051 (1981)

51 P Goddard and D I Olive: in Vertex Operators in Mathematıcs and Physics, ed. by J Lepowsky, S Mandelstam and I Singer (Springer Verlag, Heidelberg, 1984); Int.J.Mod.Phys. A1, 303 (1986)

52 B. Zumino. in Anomalies, Cocycles and Schwinger Terms ed. by W.A. Bardeen and A R. White (World Scientıfic, Singapore, 1986)

53 C. Saclioglu: preprint CERN-TH 4854 (1987)

54 I.B. Frenkel and V.G. Kac: Inv Math. 62, 23 (1980), G. Segal. Comm Math.Phys 80, 301 (1981)

55 A.J. Feingold and I B. Frenkel. Math.Ann. 263, 87 (1983)

56 E. Cremmer, B. Julia and J Scherk- Phys.Lett 76B, 409 (1978)

57 M. Ademollo et al.: Phys.Lett. B62,105 (1976);

L. Brink and J.H. Schwarz. Nucl.Phys. B12, 285 (1977);

P Ramond and J.H Schwarz Phys
I Bars. Nucl Phys 308, $462(1988)$ 